\title{
Berechnung unelastischer Rahmen nach der Theorie 2. Ordnung
}

\section{Working Paper}

\section{Author(s):}

Aas-Jakobsen, Knut; Grenacher, Mathis

Publication date:

1973

Permanent link:

https://doi.org/10.3929/ethz-a-000674500

Rights / license:

In Copyright - Non-Commercial Use Permitted

Originally published in:

Bericht / Institut für Baustatik ETH Zürich 45 
Berechnung unelastischer Rahmen nach der Theorie 2. Ordnung

Knut Aas-Jakobsen

Mathis Grenacher 


\section{Berechnung unelastischer Rahmen nach der Theorie 2. Ordnung}

von

Lic. techn. Knut Aas-Jakobsen

Dipl. Ing. Mathis Grenacher

Institut für Baustatik

Eidgenössische Technische Hochschule Zürich 
1. EINLEITUNG

1.1 Problemstellung

1.2 Generelles Vorgehen bei der Berechnung von Rahmentragwerken

2. PROBLEME BEI DER BERECHNUNG VON STAHLBETONRAHMEN

2.1 Berechnung von elastischen Stabtragwerken mit der Methode der finiten Elemente

2.2 Berechnung der effektiven Biegesteifigkeit $\overline{E I}$

2.3 Steuerung des Rechenvorganges

3. BESCHRIEB DES PROGRAMMES

3.I Genereller Aufbau des Programmes

3.2 Beschrieb der einzelnen Programmteile

4. RECHENBEISPIELE

BEZEICHNUNGEN

LITERATUR

28

VERDANKUNGEN

ZUSAMMENFASSUNG - RESUME - SUMMARY

ANHANG

1. Eingabe der Daten

2. Beispiel einer Dateneingabe

3. Output der Resultate 


\subsection{Problemstellung}

In Erweiterung des Forschungsprojektes des Instituts für Baustatik, Abt. Massivbau, ETH-Z, uber die Tragfahigkeit von Stahlbetonstützen unter Berücksichtigung von unelastischen Verformungen wird das Verhalten von Stahlbetonrahmen studiert. In einem ersten Schritt wird ein Rechenprogramm entwickelt zum Studium von schlanken Rahmentragwerken. Das Programm muss einen vielseitigen Anwendungsbereich in bezug auf die Geometrie und das Materialverhalten des Tragwerkes ermöglichen. Es müssen somit die folgenden Einflüsse berücksichtigt werden:

- Die Gleichgewichtsbedingungen werden am deformierten System formuliert (Theorie 2. Ordnung).

- Dem nicht-linearen Verformungsverhalten unter Kurz- und Langzeitlasten wird Rechnung getragen.

- Eine beliebige Lastgeschichte kann berücksichtigt werden.

Dabei werden die folgenden, grundlegenden Annahmen getroffen:

- Ebene Querschnitte bleiben eben.

- Der Einfluss der Querkraft auf die Durchbiegungen wird vernachlăssigt.

- Die Durchbiegungen bleiben klein im Verhältnis zu den Spannweiten.

- Es werden nur ebene Tragwerke berechnet unter Belastungen in der Tragwerksebene.

Die Anwendung dieses Rechenprogrammes wird am Beispiel von Stahlbetontragwerken gezeigt.

\subsection{Generelles Vorgehen bei der Berechnung von Rahmentragwerken}

Um ein möglichst vielseitiges Rechenprogramm entwickeln zu können und die Nichtlinearităt des Materials zu berücksichtigen, wird das ganze Tragwerk diskretisiert (Bild 1).

Das Tragwerk wird unterteilt in Stabe (Balken und Stützen). Damit ist eine Lösung fur beliebige ebene Rahmentragwerke möglich. Die einzelnen Stäbe werden ihrerseits unterteilt in eine Anzahl von Elementen. Ueber die Läne der Elemente wird eine konstante effektive Steifigkeit $\overline{E I}$ vorausgesetzt, was nur für kleine Elemente vernünftig ist.

Für nichtlineares Materialverhalten kann im allgemeinen keine geschlossene Beziehung zwischen Schnittkräten und Querschnittsverformungen (d.h. für die Momenten-Normalkraft-Zeit-Krummungs-Beziehung) formuliert werden. Deshalb wird der Querschnitt in einzelne Fasern unterteilt. Die Spannung wird für jede Faser direkt aus der SpannungsDehnungs-Beziehung des entsprechenden Materials berechnet.

Das in Kap. 3 beschriebene Rechenprogramm ist auf einer sukzessiven Steifigkeits- 

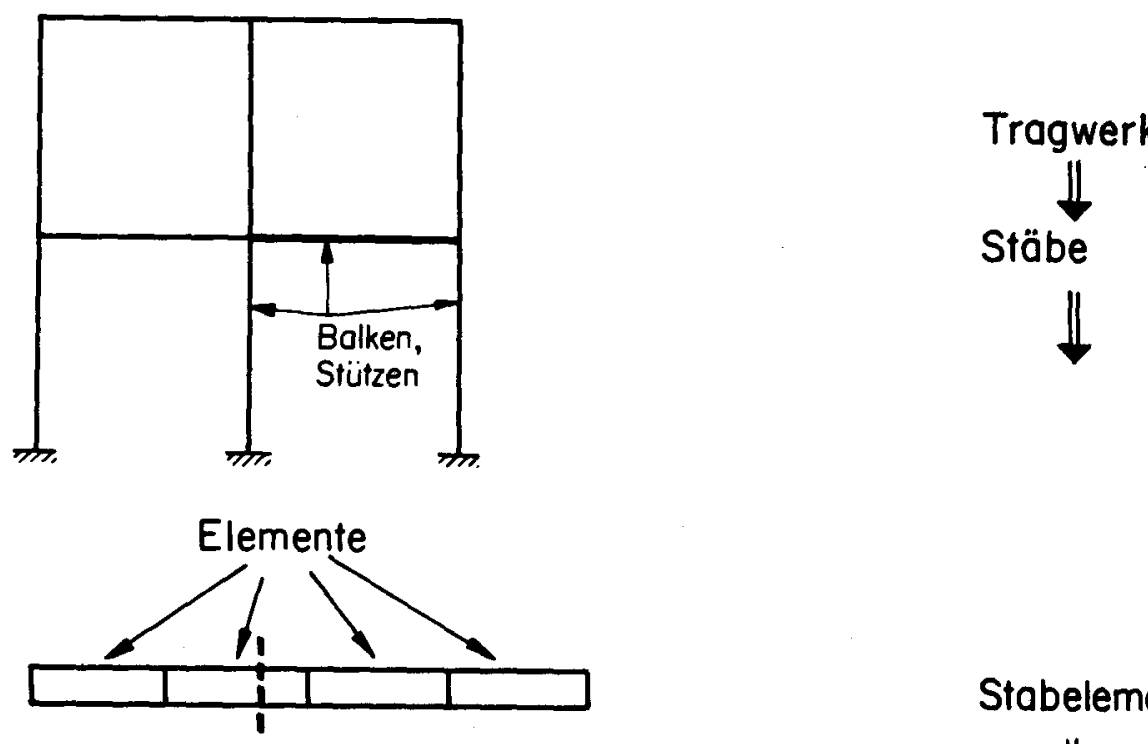

Stabelemente

Querschnitt: Dehnungen:

Sponnungen :
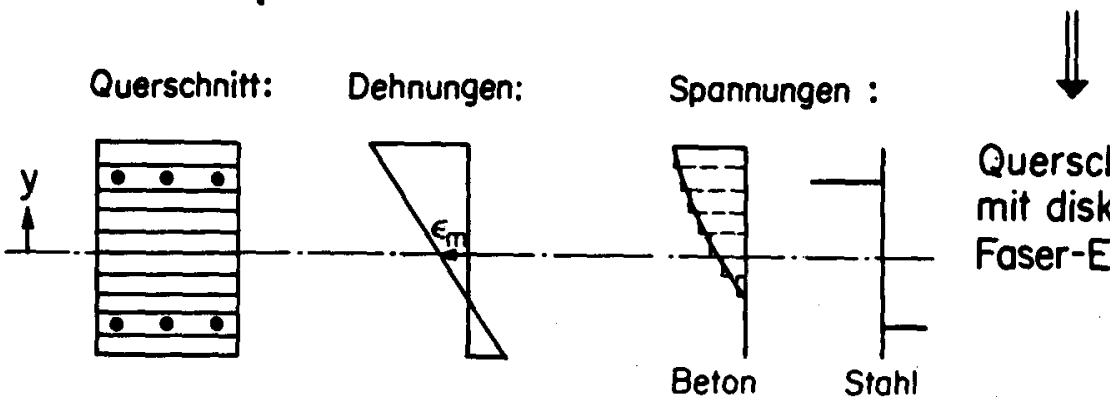

Querschnitt mit diskreten Faser-Elementen

Bild 1: Diskretisation des Tragwerks

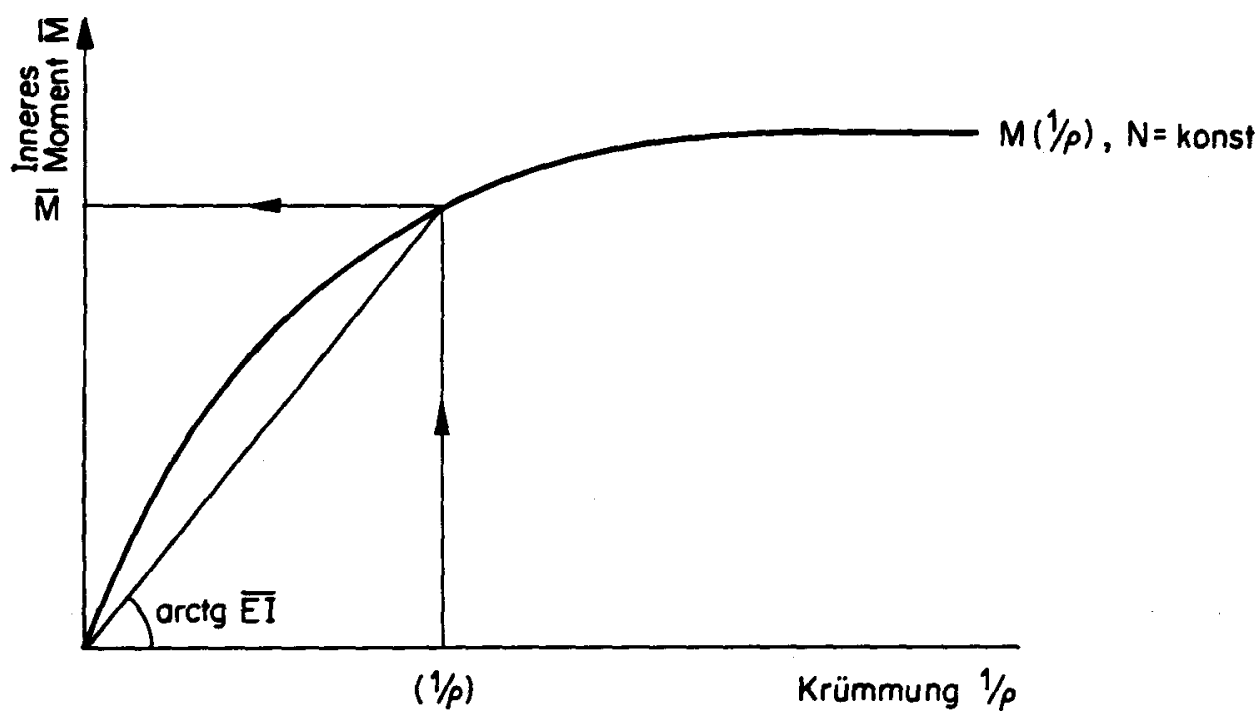

Vorgehen: $\underbrace{\overline{E I} \rightarrow M, N}_{\begin{array}{c}\text { elastische } \\ \text { Analyse }\end{array}} \rightarrow \underbrace{1 / p=M / \overline{E I} \rightarrow M}_{\begin{array}{c}\text { nicht lineare } \\ \text { Querschnittsanalyse }\end{array}} \rightarrow \overline{E I}=\bar{M} /(1 / p) ; \overline{E I} \rightarrow \cdots \cdots$

Bild 2: Berechnung der effektiven Biegesteifigkeit $\overline{E I}$ 
Approximation aufgebaut. In einem iterativen Rechenprozess wird die Biegesteifigkeit für jede Laststufe solange verändert, bis sie zu einem Gleichgewichtszustand der inneren und ausseren Krafte (d.h. Momente und Achsialkräte) fuhrt. Die einzelnen Schritte im verwendeten Rechenverfahren können wie folgt beschrieben werden:

1. Die Steifigkeiten aller Tragwerkselemente werden angenommen.

2. Für eine Belastung werden die Schnittkrăfte $M$ und $N$ sowie die Krümmung $1 / \rho$ $(=M / \overline{E I})$ für alle Elemente mit einer elastischen Analyse des Tragwerks berechnet, wobei der Einfluss 2. Ordnung mitberüksichtigt wird.

3. Für die berechnete Krümmung $1 / \rho$ und die Achsialkraft $N$ wird das innere Moment $\bar{M}$ für jedes Element unter Wahrung des achsialen Gleichgewichts ermittelt.

4. Ein besserer Wert für die effektive Steifigkeit $\overline{E I}$ kann aus dem inneren Moment $\bar{M}$ wie folgt berechnet werden:

$\overline{E I}=\bar{M} /(1 / p)$

5. Eine weitere elastische Analyse wird mit der neuen Steifigkeit durchgefunrt (Pt.2) und das Verfahren wird wiederholt bis ein $z u$ definierendes Konvergenzkriterium erfullt ist (Bild 2).

Die zwei wichtigsten Schritte in der Ermittlung des Verformungsverhaltens von schlanken Rahmentragwerken mit nichtlinearen Materialeigenschaften sind in Kapitel 2 beschrieben. Es sind dies:

- Die elastische Analyse (Kap. 2.1)

- Die Momenten-Krummungs-Beziehungen unter Berücksichtigung des nichtlinearen Materialverhaltens (Kap. 2.2).

Eine Schwierigkeit beim oben beschriebenen Verfahren ergibt sich bei schlanken Rahmentragwerken mit nichtlinearem.Materialverhalten aus der Tatsache, dass die Traglast einem labilen Gleichgewichtszustand entsprechen kann. Dieser Zustand ist ersichtlich aus Bild 3 und der Punkt der maximalen Last wird als Stabilitätsgrenze bezeichnet. Wenn die Last schrittweise erhbht wird, ist es schwierig, im kritischen Bereich der Stabilitătsgrenze einen Gleichgewichtszustand zu finden, und die Konvergenz des iterativen Rechenprozesses ist schlecht. Keine Schwierigkeiten ergeben sich jedoch, wenn an Stelle der Last die Verformung an einem Punkt des Tragwerkes schrittweise erhöht wird und die dazugehörende Last berechnet wird. 


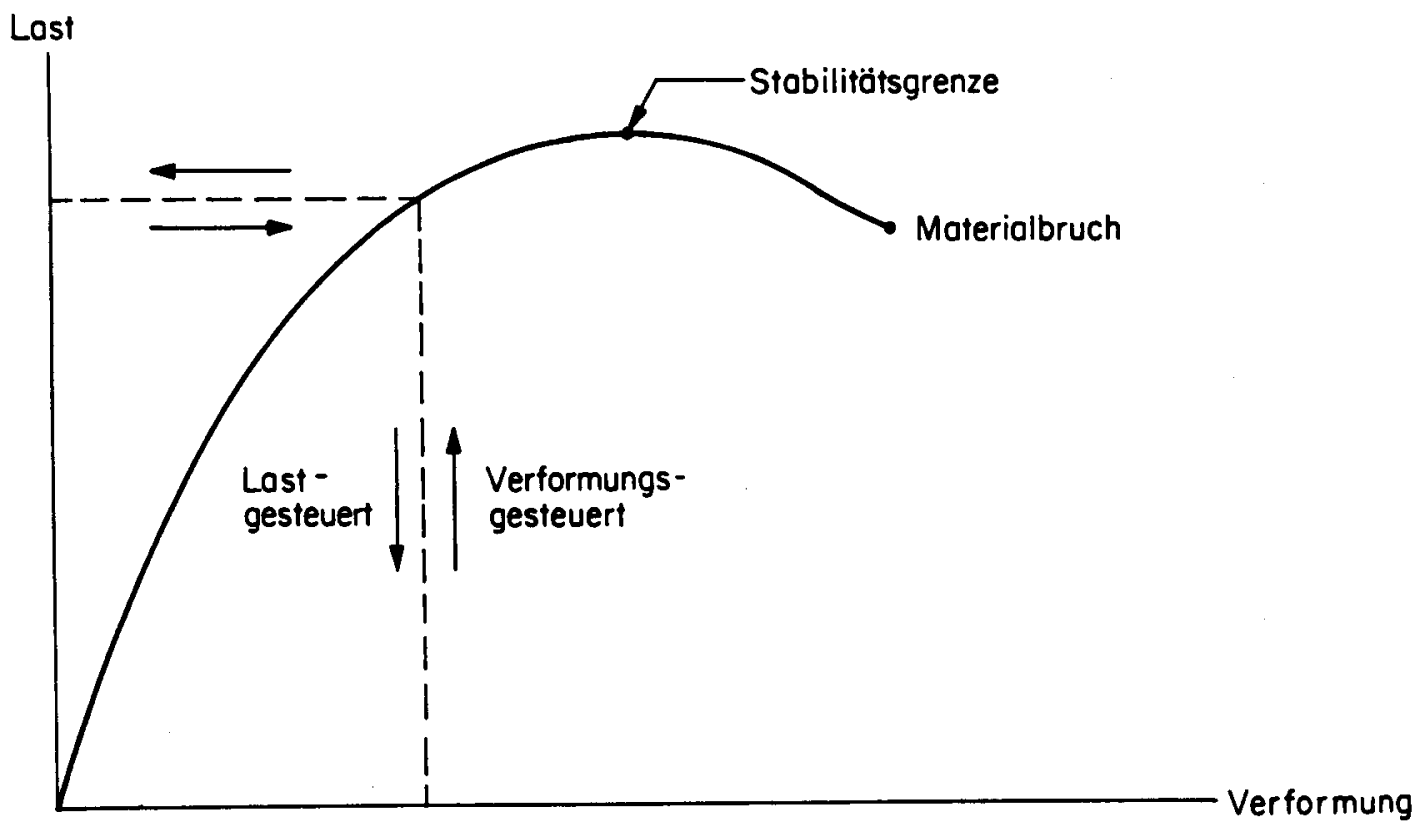

Bild 3: Versagen von schlanken Rahmentragwerken mit unelastischem Materialverhalten 


\subsection{Berechnung von elastischen Stabtragwerken mit der Methode der finiten Elemente}

Die Methode der finiten Elemente ist andernorts mehrfach beschrieben worden (z.B. [1], [2], [3]). An dieser Stelle sollen nur einige grundsätzliche Ideen und deren Anwendung im Rechenprogramm gegeben werden. Das Programm ist in Kap. 3 beschrieben.

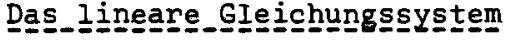

Wie bereits in der Einleitung erwahnt, wird das Rahmentragwerk in Elemente unterteilt. Diese Elemente sind an ihren Enden in den sogenannten Knotenpunkten miteinander verbunden. Die Verschiebungen dieser Knotenpunkte sind die grundlegenden Unbekannten bei der Berechnung des Tragwerkes. In jedem Knoten gibt es drei mögliche aussere Lasten, zwei Verschiebungen und eine Verdrehung (Bild 4).

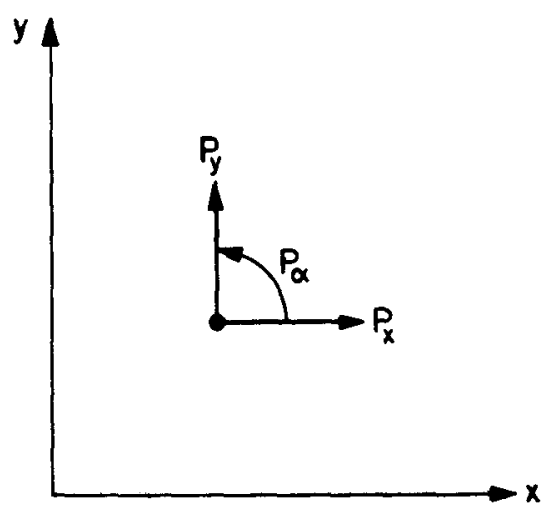

Aussere Lasten

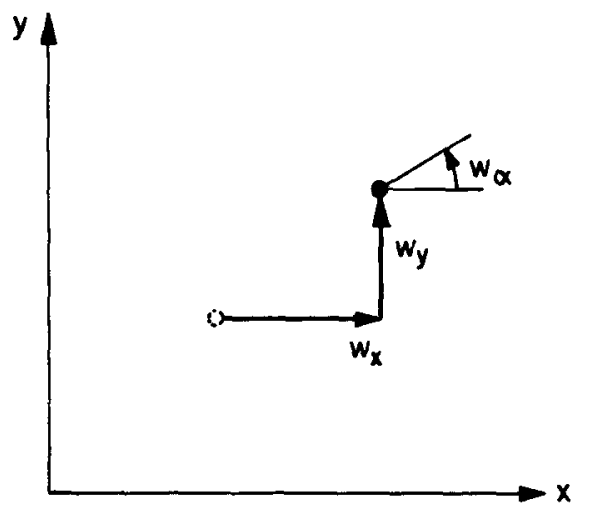

Knotenverschiebungen

\section{Bild 4: Knotenverschiebungen $\{w\}$ und äussere Lasten $\{P\}$}

Die Beziehungen zwischen den Knotenverschiebungen $\{w\}$ und den Knotenkräften $\{P\}$ fuhren zu einem Iinearen Gleichungssystem von folgender Form:

$$
[K]\{w\}=\{P\}
$$

wobei [K] die globale Steifigkeitsmatrix des Tragwerks ist.

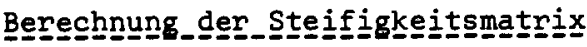

Die Ermittlung der globalen Steifigkeitsmatrix [K] eines Tragwerkes erfordert zuerst die Berechnung der Steifigkeitsmatrix fur jedes einzelne Element in irgendeinem günstigen lokalen Koordinatensystem. Um auch den Einfluss 2. Ordnung zu berücksichtigen, kann die Gleichung ( 1 ) wie folgt erweitert werden: 


$$
\left(\left[K_{1}\right]-\left[K_{2}\right]\right)\{w\}=\{P\}
$$

wobei $\left[K_{1}\right]$ die Steifigkeitsmatrix nach Theorie 1 . Ordnung ist

und $\left[K_{2}\right]$ die Ergănzung der Steifigkeitsmatrix infolge der Theorie 2. Ordnung ist. $\left[K_{2}\right]$ wird oft auch als geometrische Steifigkeitsmatrix bezeichnet.

In Anwendung des 1. Theorems von Castigliano kann die lokale Steifigkeitsmatrix eines Elementes aus der Formänderungsenergie $U$ abgeleitet werden. Die Steifigkeitskoeffizienten $k_{i j}$ berechnen sich zu:

$$
k_{i j}=\frac{\partial^{2} U}{\partial w_{i} \partial w_{j}}
$$

Die Formänderungsenergie U kann in Funktion der Verschiebungen fur prismatische Stabe wie folgt ausgedrückt werden:

$$
U=\frac{1}{2} E I \int_{L}\left(\frac{d^{2} w_{y}}{d x^{2}}\right)^{2} d x+\frac{1}{2} E F \int_{L}\left(\frac{d w}{d x}\right)^{2} d x+\frac{1}{2} \int_{L} N\left(\frac{d w y}{d x}\right)^{2} d x
$$

Die beiden ersten Terme entsprechen den bekannten Ausdrücken fü die Formänderungsenergie bei Biegung resp. achsialer Verformung. Der letzte Term entspricht der Energie, die $\mathrm{N}$ bei der Verkürzung infolge Verbiegung des Elementes beitrăgt. Dies ist der Einfluss 2. Ordnung.

Um die Formänderungsenergie berechnen zu konnen, muss das Verschiebungsfeld $w_{x}(x)$ und $w_{y}(x)$ in Funktion der lokalen Knotenverschiebungen \{w\} gegeben sein. Diese Beziehung für die beiden Verschiebungen kann erfasst werden durch den Ansatz eines Polynoms von der gleichen Ordnung wie Knotenverschiebungen pro Element vorhanden sind. Dies ergibt ein Polynom 1 . Ordnung fü die Verschiebung $w_{x}(x)$ entlang der Elementachse und ein Polynom 3 . Ordnung fur die Verschiebung $w_{y}(x)$ normal zur Elementachse. Ausgedrückt in Funktion der Knotenverschiebungen in einem lokalen Koordinatensystem mit der $x$-Achse entlang der Elementachse und der y-Achse normal dazu ergeben sich die verschiebungen $w_{x}$ und $w_{y} z u$ :

$$
\begin{aligned}
w_{x} & =w_{x}^{i}+\left(w_{x}^{j}-w_{x}^{i}\right) \xi \\
w_{y} & =\left(1-3 \xi^{2}+2 \xi^{3}\right) w_{y}^{i}+L\left(\xi-2 \xi^{2}+\xi^{3}\right) w_{\alpha}^{i}+\left(3 \xi^{2}-2 \xi^{3}\right) w_{y}^{j}+ \\
& +L\left(-\xi^{2}+\xi^{3}\right) w_{\alpha}^{j}
\end{aligned}
$$

wobei $\xi=\frac{x}{L}$

Durch Einsetzen der Ausdrücke (4) für die Verschiebungen in die Gleichung (3) ergibt sich aus (2) die Elementsteifigkeitsmatrix [K] lokal

Um die Elementsteifigkeitsmatrizen zu einer Systemsteifigkeitsmatrix zusammenfügen zu konnen, müssen die lokalen Matrizen der Elemente auf ein globales Koordinatensystem bezogen werden. Mit einer Rotationsmatrix $[R]_{6 \times 6}$, die abhangig ist vom entsprechenden Winkel a des Elementes, ergibt sich die globale Steifigkeitsmatrix zu: 


$$
[K]=[R][K]_{\text {lokal }}[R]^{T}
$$

Mit den folgenden Abkürzungen:

$$
s=\sin \alpha \quad c=\cos \alpha
$$

wird:

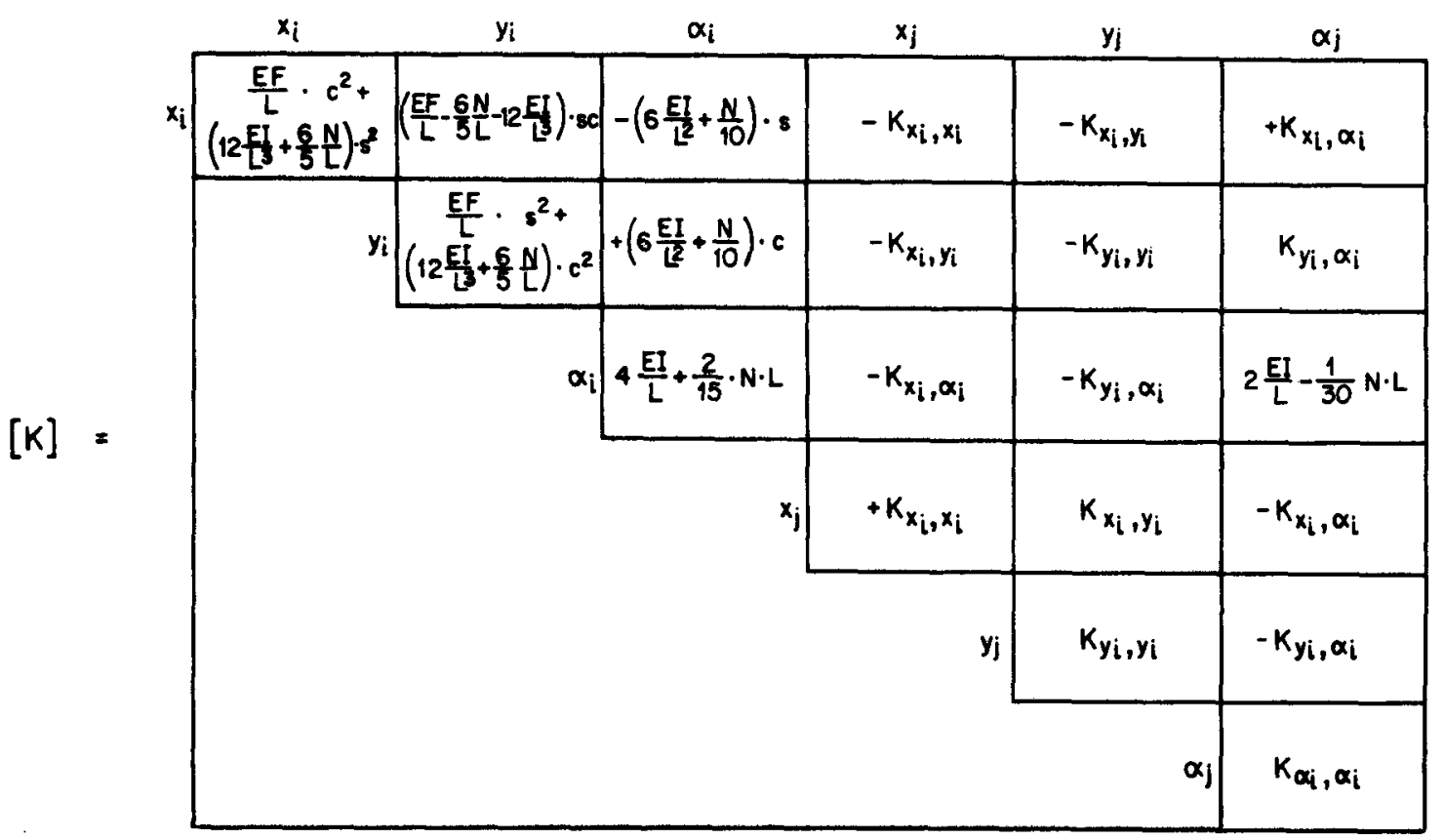

Un die Steifigkeitsmatrix [K] zu berechnen, muss allerdings für jedes Element die Normalkraft $\mathrm{N}$ bekannt sein. Dies ist normalerweise nicht der Fall, sodass ein iterativer Rechenprozess, der aber sehr schnell konvergiert, notwendig ist. [K] wird für die Normalkräfte aus dem vorhergehenden Iterationsschritt berechnet.

\section{Geometris_che Randbedingungen und_gegebene Versschiebungen}

Da die Verschiebungen die Unbekannten des linearen Gleichungssystems (1) sind, vereinfachen geometrische Randbedingungen, die gegebenen Knotenverschiebungen entsprechen, das Gleichungssystem. Ganz allgemein kann eine solche Vereinfachung infolge einer gegebenen Knotenverschiebung $w_{i}$ durch die folgenden Manipulationen berücksichtigt werden:

1. Alle Glieder der $i$-ten Kolonne des Gleichungssystems (d.h. $\left.k_{j, i} \cdot w_{i}\right)$ werden auf die rechte Seite des Gleichheitszeichens gebracht.

2. Die Koeffizienten der i-ten Kolonne und der i-ten Zeile der Steifigkeitsmatrix [K] werden gleich 0 gesetzt.

3. $K_{i, i}$ wird gleich 1 und $P_{i}$ gleich $w_{i}$ gesetzt. 
Damit vereinfacht sich das Gleichungssystem (1) - die i-te Gleichung wird trivial wie folgt:

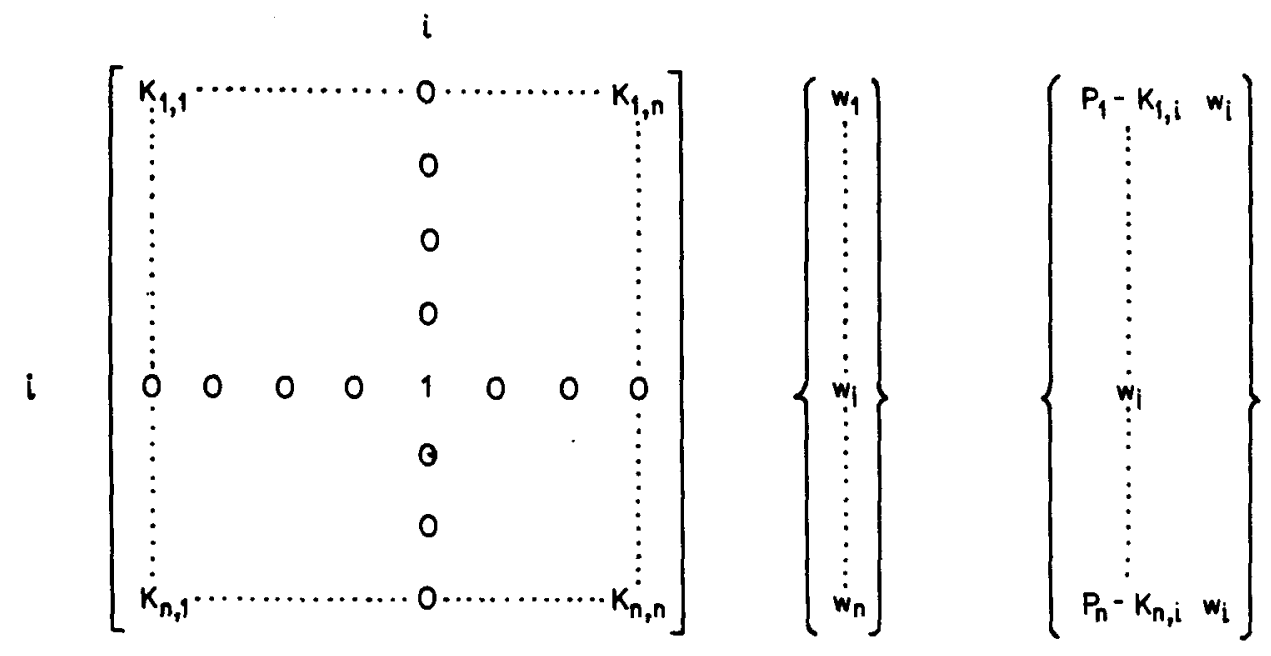

(6)

Wenn die Reaktionen $\mathrm{RE}_{i}$ berechnet werden sollen, so müssen die Koeffizienten der $i-t e n$ Zeile von [K] gespeichert werden. Nach der Berechnung der Verschiebungen $\{w\}$ können die Reaktionen wie folgt berechnet werden:

$$
R E_{i}=\sum_{j=1}^{n} k_{i j} \cdot w_{j}
$$

\section{Lösung_des_Gleichungssystems_(6)_und_Berechnung_der_Elementendkräafte}

Es handelt sich um ein lineares Gleichungssystem, dessen Koeffizientenmatrix im allgemeinen bandförmig ist. Aus Symmetriegründen muss nur die Hälfte des Bandes gespeichert werden. Die Auflösung des Gleichungssystems geschieht nach dem Gauss'schen Algorithmus.

Mit den ermittelten globalen Verschiebungen $\{w\}$ lassen sich die lokalen Elementendkräfte für ein Element $i-j$ wie folgt berechnen:

$$
\left\{\begin{array}{c}
N_{i} \\
Q_{i} \\
M_{i} \\
N_{j} \\
Q_{j} \\
M_{j}
\end{array}\right\} \quad=[K]_{\text {lokal }}[R]^{T}\{w\}
$$

wobei sich unter Verwendung derselben Abkürzungen wie bei der globalen Steifigkeitsmatrix $[K]$ auf Seite 7 der folgende Ausdruck für $[K]_{\text {lokal }} \cdot[R]^{T}$ ergibt: 


$\left[\begin{array}{|c|c|c|c|c|c|}\hline \frac{E F}{L} c & \frac{E F}{L} s & 0 & -\frac{E F}{L} c & -\frac{E F}{L} \cdot & 0 \\ \hline-\left(12 \frac{E I}{L^{3}}+\frac{6}{5} \frac{N}{L}\right) s & +\left(12 \frac{E I}{L^{3}}+\frac{6}{5} \frac{N}{L}\right) c & +6 \frac{E I}{L^{2}}+\frac{N}{10} & \left(12 \frac{E I}{L^{3}}+\frac{6}{5} \frac{N}{L}\right) s & -\left(12 \frac{E I}{L^{3}}+\frac{6}{5} \frac{N}{L}\right) c & +\left(6 \frac{E I}{L^{2}}+\frac{N}{10}\right) \\ \hline-\left(6 \frac{E I}{L^{2}}+\frac{N}{10}\right) s & +\left(6 \frac{E I}{L^{2}}+\frac{N}{10}\right) c & 4 \frac{E I}{L}+2 \frac{N L}{15} & +\left(6 \frac{E I}{L^{2}}+\frac{N}{10}\right) s & -\left(6 \frac{E I}{L^{2}}+\frac{N}{10}\right) c & 2 \frac{E I}{L}-\frac{N L}{30} \\ \hline-\frac{E F}{L} c & -\frac{E F}{L} s & 0 & \frac{E F}{L} c & \frac{E F}{L} s & 0 \\ \hline\left(12 \frac{E I}{L^{3}}+\frac{6}{5} \frac{N}{L}\right) s-\left(12 \frac{E I}{L^{3}}+\frac{6}{5} \frac{N}{L}\right) c & -\left(6 \frac{E I}{L^{2}}+\frac{N}{10}\right) & -\left(12 \frac{E I}{L^{3}}+\frac{6}{5} \frac{N}{L}\right) s & +\left(12 \frac{E I}{L}+\frac{6}{5} \frac{N}{L}\right) c & -\left(6 \frac{E I}{L^{2}}+\frac{N}{10}\right) \\ \hline-\left(6 \frac{E I}{L^{3}}+\frac{N}{10}\right) & +\left(6 \frac{E I}{L^{2}}+\frac{N}{10}\right) c & 2 \frac{E I}{L}-\frac{N L}{30} & +\left(6 \frac{E I}{L^{2}}+\frac{N}{10}\right) s & -\left(6 \frac{E I}{L^{2}}+\frac{N}{10}\right) c & 4 \frac{E I}{L}+2 \frac{N L}{15} \\ \hline\end{array}\right.$

Die Vorzeichenkonvention ist dieselbe wie für die äusseren Lasten (Bild 4).

Die Elementendkräfte könnten auch aus den Ableitungen der berechneten Verschiebungen ermittelt werden. Wegen der verwendeten Verschiebungsansätze wäre dabei aber das Gleichgewicht unter Umständen nicht exakt erfüllt.

\section{$\underline{2.2 \text { Berechnung der effektiven Biegesteifigkeit } \overline{E I}}$}

\section{Generellies Vorgehen}

Wie bereits in der Einleitung beschrieben, werden für gegebene Lasten die Momente $M$ und die Normalkraft $\mathrm{N}$ in jedem Element in einer elastischen Analyse berechnet. Die dazugehörende Krümmung $1 / p$ ist:

$$
1 / p=M / \overline{E I}
$$

Zu dieser Krümung I/D kann für unelastisches Materialverhalten das dazugehörende innere Moment $\bar{M}$ unter Berücksichtigung der Normalkraft $N$ berechnet werden (Bild 2 ). Mit diesem inneren Moment $\bar{M}$ ergibt sich eine neue Steifigkeit $\overline{E I}$ :

$$
\overline{E I}=\bar{M} /(1 / 0)
$$

Damit ist ein neuer Iterationsschritt moglich. Dieses Iterationsverfahren wird angewendet bis die Biegesteifigkeiten $\overline{E I}$ in allen Elementen mit gegebener Genauigkeit konvergieren. Bei diesem Verfahren wird die Steifigkeit $\overline{E I}$ als konstant uber die Elementlănge angenommen. Normalerweise wird die Steifigkeit in der Mitte eines jeden Elementes bestimmt. 


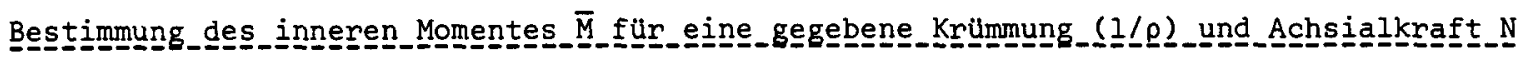

Die inneren Krafte werden bestimnt durch eine Integration der Spannungen uber die Querschnittsfläche:

$$
\begin{aligned}
& \bar{N}=\sum \sigma \Delta \mathrm{F} \\
& \bar{M}=\Sigma \sigma \cdot y \Delta F
\end{aligned}
$$

Die Spannungen sind abhängig von den Dehnungen, die sich unter der Annahme einer linearen Dehnungsverteilung wie folgt berechnen lassen:

$$
\varepsilon=\varepsilon_{m}+(I / \rho) y
$$

wobei $\varepsilon_{\mathrm{m}}=$ Dehnung in Querschnittsmitte

$$
1 / \rho=\text { Krümmung }
$$

In Gleichung (12) ist nur die Dehnung in Querschnittsmitte $\varepsilon_{\mathrm{m}}$ unbekannt. Diese Position der Dehnungsebene wird in einem iterativen Rechenprozess bestimmt, bei dem die Lage der Dehnungsebene so lange verăndert wird, bis die resultierende Normalkraft dem ausseren $N$ entspricht. Die iterative Bestimmung von $\varepsilon_{\mathrm{m}}$ aus dem achsialen Gleichgewicht kann beschleunigt werden durch verschiedene Methoden wie Newton-Raphsons oder andere. In diesem Programm wird ein Langrange Polynom verwendet, wie es in [4] von Aitken beschrieben worden ist.

In den einzelnen Fasern des Querschnitts muss nur die Spannungs-Dehnungs-Zeitbeziehung der Materialien bekannt sein.

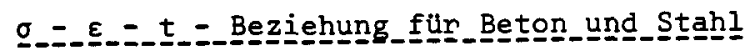

Um die inneren Kräfte für eine bestimmte Dehnungsverteilung bestimmen zu können, müssen die Spannungs-Dehnungs-Zeit-Beziehungen fü die Materialien formuliert sein. Im folgenden werden die einfachen Beziehungen für Beton und Armierungsstahl beschrieben, wie sie im Programm verwendet werden. Sie sind in einer einzigen Subroutine programmiert und können deshalb ohne grossen Aufwand durch andere Beziehungen ersetzt werden.

Die grundlegende Beziehung zwischen Spannungen und Dehnungen wird für Beton und für Stahl wie folgt formuliert:

$$
\sigma=E_{0}\left(\varepsilon-\varepsilon_{f}\right)
$$

wobei $E_{0}=$ initialer Elastizitätsmodul

$\varepsilon=$ totale Dehnung

$\varepsilon_{f}=$ "plastische" Dehnung zum betrachteten Zeitpunkt

Die Spannung, wie sie mit (13) berechnet wird, muss limitiert werden durch das $\sigma-\varepsilon-$ Diagramm für eine Kurzzeitbelastung bis zum Bruch. Diese maximal möglichen Spannungen sind gegeben in Bild 5 . 

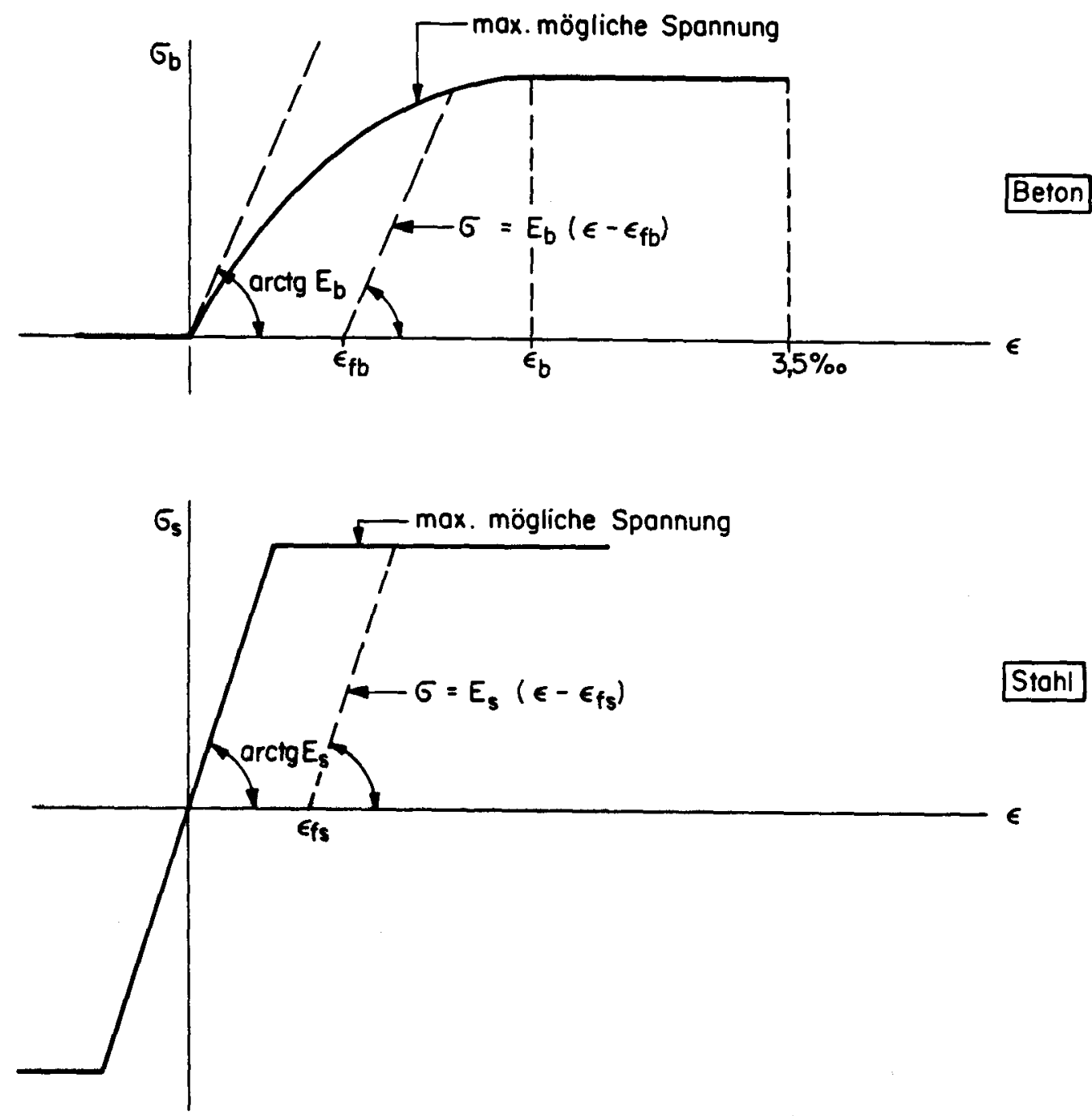

\section{Bild 5: Spannungs-Dehnungs-Beziehungen}

Für Stahl ist die Spannung durch die Streck- oder Fliessgrenze $\sigma_{0,2}$ beschränkt:

$$
\left|\sigma_{s, \max }\right| \leq\left|\sigma_{0,2}\right|
$$

Für Beton ist die Spannung beschränkt durch:

$$
\begin{aligned}
& \sigma_{b, \max }=0 \text { für } \varepsilon \leq 0 \text { (Zugdehnung) } \\
& \sigma_{b, \max }=\beta_{B r}\left(2 \varepsilon / \varepsilon_{b}-\left(\varepsilon / \varepsilon_{b}\right)^{2}\right) \text { für } 0 \leq \varepsilon \leq \varepsilon_{b} \\
& \sigma_{b, \max }=B_{B r} \text { für } \varepsilon_{b} \leq \varepsilon \leq 3,5 \text { 80 }
\end{aligned}
$$

Wird nun zu einem Zeitpunkt $t+\Delta t$ die Spannung $\sigma$ fur die totale Dehnung $\varepsilon$ gesucht, so muss zuerst, um die Gleichung (13) verwenden zu können, $\varepsilon_{f}$ berechnet werden. Diese Berechnung der "plastischen" Dehnung soll im folgenden beschrieben werden.

Das Verhalten des Armierungsstahles wird als zeitunabhängig angenommen, sodass die Spannung zur Zeit $t+\Delta t$ direkt mit Gleichung (13) bestimmt werden kann. Die "pla- 
stische" Dehnung $\varepsilon_{f s}$ wird nur verändert, wenn die Spannung o die Fliessspannung (14a) uberschreitet.

Die "plastische" Dehnung des Betons ergibt sich aus drei verschiedenen Einflüssen:

- Die "plastische" Dehnung bei Kurzzeitbelastung (Bild 5).

Dieser Anteil an $\varepsilon_{f}$ ist unabhängig von der Zeit.

- Die "plastische" Dehnung infolge Schwinden. Dieser Anteil ist unabhängig von der Spannung. Die Vergrösserung der Schwinddehnung $\Delta \varepsilon_{f 1}$ im Zeitintervall $\Delta t$ wird wie folgt angenommen:

$$
\Delta \varepsilon_{f 1}=\varepsilon_{\operatorname{sch}, \infty}\left(\frac{t+\Delta t}{t_{1 / 2}+t+\Delta t}-\frac{t}{t_{1 / 2}+t}\right)
$$

wobei:

$$
\begin{aligned}
& \varepsilon_{\text {sch, }, \infty}=\text { Endschwinddehnung } \\
& t_{y 2}=\text { Zeitpunkt, bei dem } \varepsilon_{\text {sch }}=1 / 2 \varepsilon_{\text {sch, } \infty}
\end{aligned}
$$

Der Ansatz (15) entspricht einer hyperbolischen Vergrösserung mit der Zeit. Durch geeignete Wahl von $\varepsilon_{s c h, \infty}$ und $t_{y_{2}}$ kann dieser Ansatz gut einer gemessenen Kurve angeglichen werden.

- Der dritte Anteil an der "plastischen" Dehnung ist die Kriechdehnung. Die Vergrösserung der Kriechdehnung $\Delta \varepsilon_{f 2}$ in Zeitintervall $\Delta t$ wird wie folgt angenommen:

$$
\Delta \varepsilon_{f 2}=\varepsilon_{k r, \infty}\left(\frac{t+\Delta t}{t_{1 / 2}+t+\Delta t}-\frac{t}{t_{1 / 2}+t}\right)
$$

Dieser Ansatz (16) ist ähnlich aufgebaut wie der Ansatz (15) für das Schwinden. $\varepsilon_{k r, \infty}$ ist die Endkriechdehnung, die abhängig ist von der Spannung und sich mit der Kriechzahl 0 wie folgt ausdrücken lasst:

$$
\varepsilon_{\mathrm{kr}, \infty}=\varepsilon_{0}
$$

$\varepsilon_{0}$ ist die Dehnung infolge einer Kurzzeitbelastung und berechnet sich aus (14b) zu

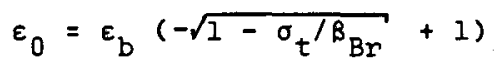

Vereinfachend wird die Spannung $\sigma_{t}$ zur Zeit $t$ verwendet, um die Kriechdehnung im Zeitintervall $\Delta t$ zu ermitteln. Die Endkriechdehnung $\varepsilon_{k r, \infty}$ unter konstanter Spannung ist nach Ansatz (17) proportional zur Dehnung zur Zeit $t=0$. Der Verlauf der Kriechdehnungen uber die Zeit $t$ kann in (16) resp. (17) durch geeignete Wahl von o und $t_{1 / 2}$ einer gemessenen Kurve angepasst werden.

Das oben beschriebene Verfahren für die Berechnung der Kriechdehnungen unter variabler Spannung entspricht der "rate of creep" Methode. 
Mit den beschriebenen Ansätzen kann die Vergrösserung der "plastischen" Dehnungen berechnet werden und damit wird die Spannung zur Zeit $t+\Delta t$ mit Gleichung (13) ermittelt. Nach jeder Spannungsberechnung muss die Kontrolle gemacht werden, ob die Grenzspannungen (14) nicht überschritten werden.

Die totale "plastische" Dehnung zur Zeit $t+\Delta t$ ist Grundlage für den nächsten Rechengang und kann aus Gleichung (13) wie folgt berechnet werden:

$$
\varepsilon_{f}=\varepsilon-\sigma_{t+\Delta t} / E_{0}
$$

Diese Gleichung gilt für Stahl wie auch für Beton.

\subsection{Steuerung des Rechenvorganges}

\section{Eingleitunng}

Mit dem in diesem Bericht beschriebenen Programm kann das Verhalten von schlanken Rahmen für eine beliebige Lastgeschichte untersucht werden. Neben konstanten Lasten können zusätzlich proportionale Lasten, die mit einem Lastfaktor $\lambda$ variiert werden, in der Rechnung berucksichtigt werden. Ein Beispiel für diese beiden Möglichkeiten ist in Bild 6 gegeben.

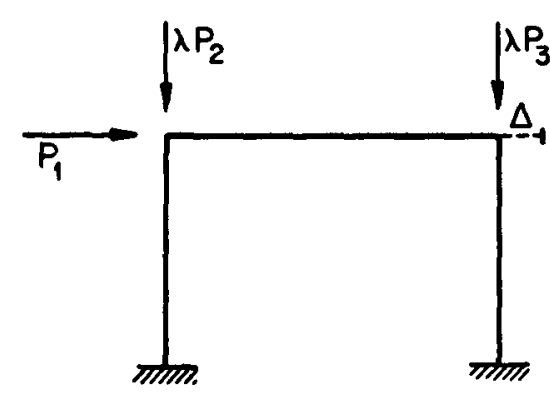

$$
P_{1} \quad=\text { konstante Lost }
$$

$P_{2}, P_{3}=$ Proportionale Lasten

$\lambda \quad=$ Gesuchter Lastfaktor

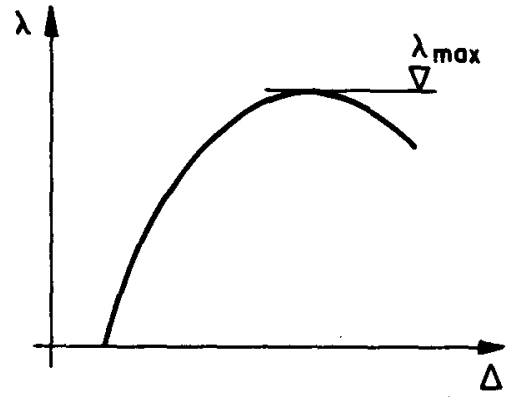

\section{Bild 6: Beispiel für einen möglichen Belastungsfall}

Wie bereits in Kap. I erwähnt, bieten sich zwei Rechenverfahren an. Einerseits kann der Lastfaktor $\lambda$ stufenweise erhöht und für jede Laststufe der entoprechende Gleichgewichtszustand ermittelt werden. Andererseits kann irgendeine Verformung kontrolliert und stufenweise erhöht werden. Dabei nimnt der zu berechnende Lastfaktor $\lambda$ bis zu einem maximalen Wert (Traglast) zu und bei weiterer Verformung wird er wieder kleiner. Diese verformungsgesteuerte Methode hat den Vorteil, dass das Tragwerk ohne Konvergenzschwierigkeiten über den kritischen Zustand hinaus berechnet werden 
kann.

Für die verformungsgesteuerte Traglastberechnung muss ein sogenannter KontrollSchnitt spezifiziert werden. In diesem Schnitt wird die Krümmung kontrolliert und stufenweise erhöht. Aus der Last-Verformungs-Beziehung (Bild 3) wird der Lastfaktor $\lambda$ für die gegebene Krümmung bestimmt. Der Kontroll-Schnitt kann beliebig gewählt werden. Die Wahl dieses Schnittes hat aber einen Einfluss auf die Konvergenz dex Rechnung. Am günstigsten ist derjenige Schnitt, in dem die Krümmung den grössten Einfluss hat auf den Lastfaktor $\lambda$. Normalerweise wird der Schnitt mit dem grössten Moment als Kontroll-Schnitt bezeichnet.

\section{Ste-ueverung}

Für das lastgesteuerte wie auch für das verformungsgesteuerte Rechenverfahren wird dieselbe elastische Analyse des Tragwerkes durchgeführt (Kap. 2.1). Für gegebene konstante Lasten werden mit den Biegesteifigkeiten und Normalkräften, die aus dem vorhergehenden Rechengang bekannt sind, für zwei Werte des Lastfaktors $\lambda$ die Momente, Normalkräfte und Krümmungen berechnet. Diese elastische Berechnung wird für $\lambda_{1}=1$ und $\lambda_{2}=2$ durchgeführt (Bild 7). Die beiden Gleichgewichtszustände für $\lambda_{1}$

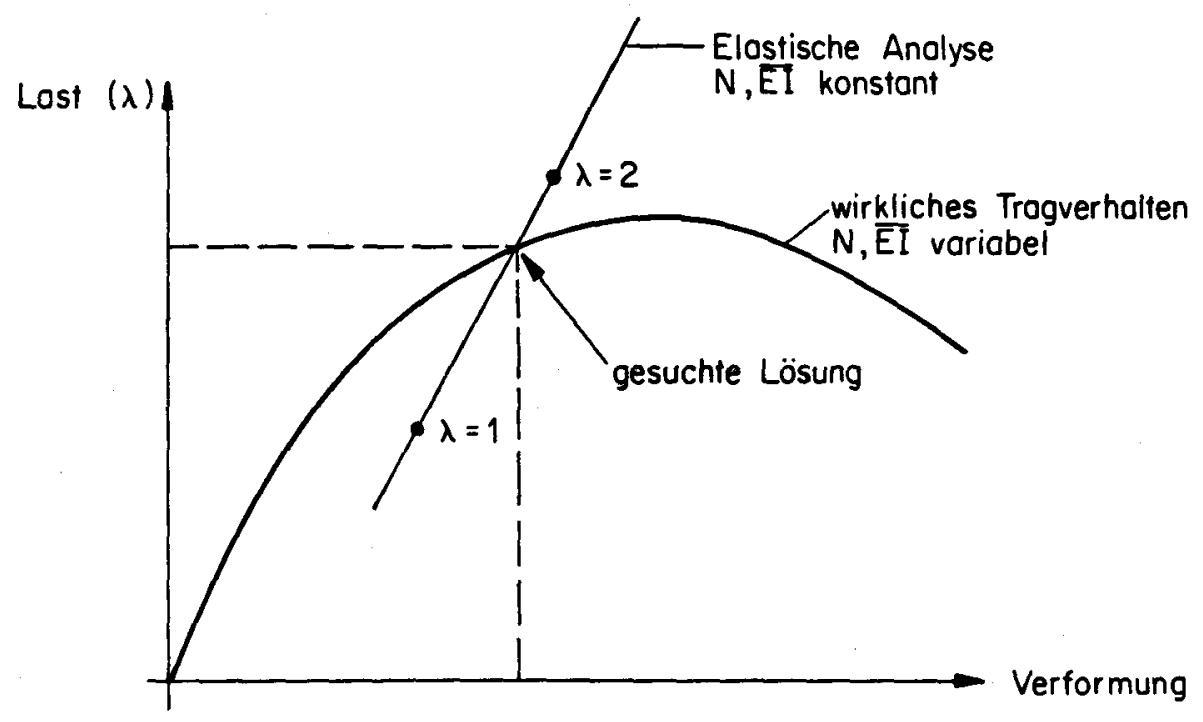

\section{Bild 7: Elastische Analyse für konstantes $\overline{E I}$ und $N$}

und $\lambda_{2}$ werden aufgrund derselben Biegesteifigkeiten und Normalkräfte ermittelt. Dies bedeutet, dass eine lineare Beziehung zwischen $\lambda$ und allen übrigen Parametern wie z.B. den Krummungen existiert. Diese Linearitat ist aus der Steifigkeitsmatrix [K] (vgl. (5)) ersichtlich. Für jede elastische Analyse können dank der linearen Beziehungen die Elementkrafte und die Krümmungen durch Interpolation wie folgt bestimmt werden:

$$
M=M_{1}+\frac{M_{2}-M_{1}}{\lambda_{2}-\lambda_{1}}\left(\lambda-\lambda_{1}\right)=M_{1}+\left(M_{2}-M_{1}\right)(\lambda-1)
$$




$$
\begin{aligned}
& N=N_{1}+\frac{N_{2}-N_{1}}{\lambda_{2}-\lambda_{1}}\left(\lambda-\lambda_{1}\right)=N_{1}+\left(N_{2}-N_{1}\right)(\lambda-1) \\
& (y \rho)=(y / \rho)_{1}+\frac{\left(y_{\rho}\right)_{2}-(y / \rho)_{1}}{\lambda_{2}-\lambda_{1}}\left(\lambda-\lambda_{1}\right)=(y \rho)_{1}+\left((y / \rho)_{2}-\left(y \lambda_{1}\right)\right)(\lambda-1)
\end{aligned}
$$

Die Indices " 1 " und " 2 " beziehen sich auf die beiden Gleichgewichtszustände für $\lambda=1$ und $\lambda=2$. Die gleiche Interpolation (20) kann auch verwendet werden, um andere Parameter wie Reaktionen und Verschiebungen zu berechnen.

Im lastgesteuerten Rechenverfahren ist der Lastfaktor $\lambda$ gegeben und die Gleichungen $(20 \mathrm{a}-\mathrm{c})$ können direkt verwendet werden. Bei bekanntem $\lambda$ könnte die elastische Analyse direkt für $\lambda$ gemacht werden, statt zuerst für $\lambda_{1}$ und $\lambda_{2}$ zu rechnen und dann zu interpolieren. Diese Vereinfachung wird aus programmtechnischen Gründen nicht gemacht, damit derselbe Rechengang auch für die Verformungssteuerung verwendet werden kann.

Im verformungsgesteuerten Verfahren ist nicht $\lambda$ sondern die Krümnung $\left(y_{p}\right)_{k s}$ in Kontroll-Schnitt gegeben. Mit der folgenden Gleichung

$$
\lambda=\lambda_{1}+\frac{\lambda_{2}-\lambda_{1}}{(y \rho)_{2}-(y / \rho)_{1}} \cdot\left((y / \rho)_{k s}-(y / \rho)_{1}\right)=1+\frac{(y \rho)_{k s}-(y \rho)_{1}}{(y \rho)_{2}-(y \rho)_{1}}
$$

muss zuerst $\lambda$ berechnet werden. Damit können für die weitere Rechnung wieder die Gleichungen $(20 \mathrm{a}-\mathrm{c})$ verwendet werden.

Mit den aufgrund dieser elastischen Berechnung ermittelten.Elementkräften und Krümmungen werden verbesserte Steifigkeiten bestimmt (Kap. 2.2). Damit wird die elastische Analyse wiederholt. Dieser Rechenprozess ist in Bild 8 dargestellt. Er wird durchgefuhrt bis die Steifigkeiten aller Elemente konvergieren.

Im verformungsgesteuerten Rechenverfahren wird, wie bereits erwähnt, die Krümmung kontrolliert und nicht irgendeine Verschiebung. Das "krümmungsgesteuerte" Verfahren hat den Vorteil, dass die Vergrösserung der Krümmung $\Delta(1 / \rho)$ für jeden Rechenschritt meist unabhängig vom zu berechnenden Tragwerk gegeben werden kann. Normalerweise wird $\Delta\left(y_{p}\right)=0.0005 / \mathrm{H}$ gesetzt, wobei $\mathrm{H}$ gleich der Querschnittshöhe im Kontroll-Schnitt ist. Würde eine Verschiebung kontrolliert, so wäre die Vergrösserung der Verschiebung abhăngig vom Tragsystem und deshalb schwierig im voraus festzulegen.

Im lastgesteuerten Rechenverfahren werden Kurzzeit- und Langzeitlasten sehr ăhlich berechnet. Anstelle der Last wird die Zeit verändert, während die gegebene Dauerlast konstant gehalten wird. Eine beliebige Last-Zeitgeschichte kann berücksichtigt werden, solange keine Gleichgewichtszustande im kritischen Bereich der Traglast berechnet werden sollen.

Etwas komplizierter ist das verformungsgesteuerte Rechenverfahren. Für Kurzzeitlasten wird die Krümung im Kontroll-Schnitt schrittweise vergrössert und für jeden Schritt wird der Lastfaktor $\lambda$ berechnet. Für Langzeitbelastung, d.h. für ein gegebenes $\lambda_{D}$ und eine gegebene Zeitdauer, wird wie bei der Kurzzeitbelastung die Krummung schrittweise erhbht. Für jeden Schritt wird die Zeit $t$ verändert bis der Lastfaktor gleich dem gegebenen Faktor $\lambda_{D}$ fur die Dauerlast ist. Die Vergrösserung der Zeitdauer $t$, die der Vergrösserung der Krümung entspricht, muss iterativ bestimmt werden. Diese 


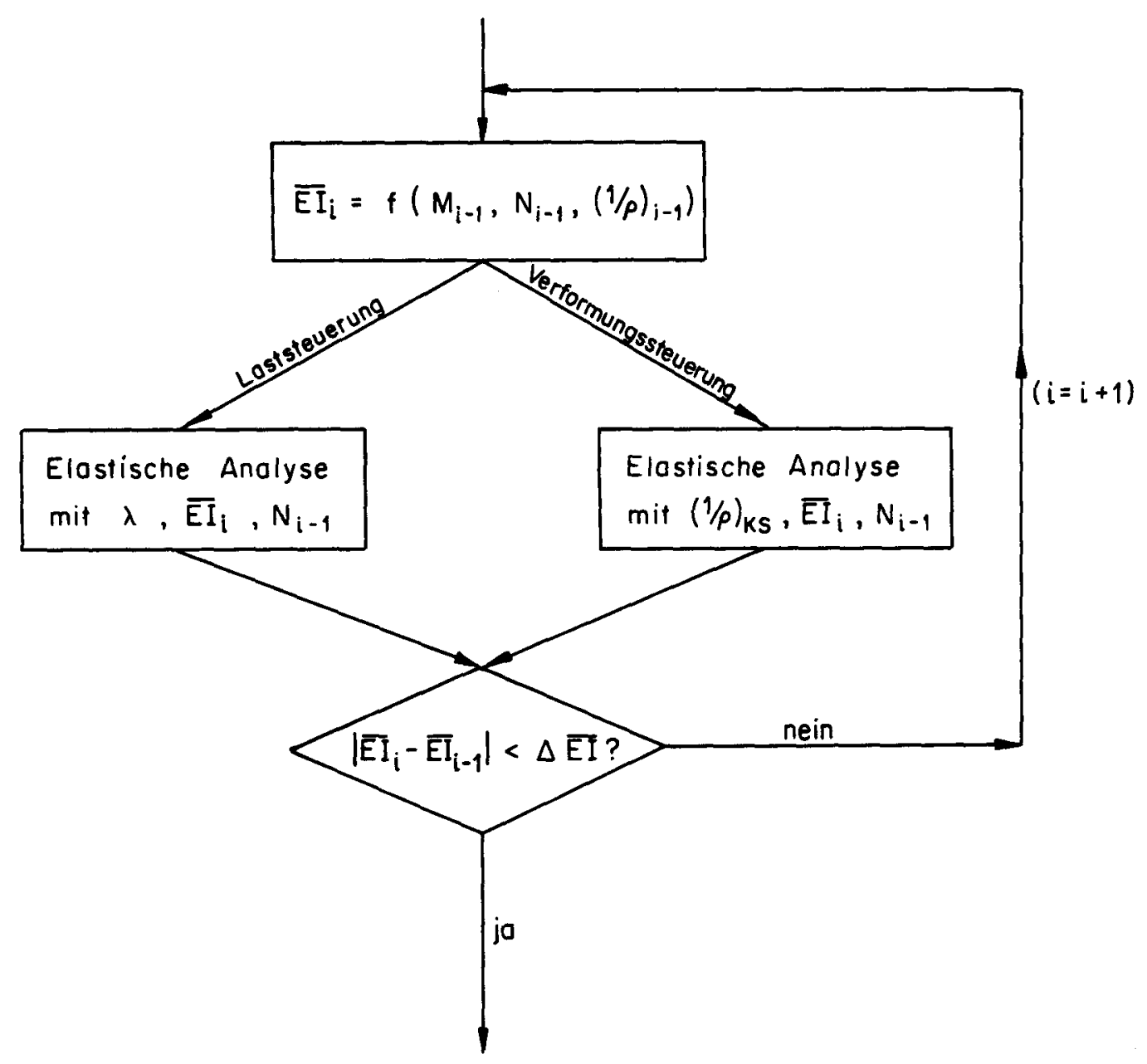

\section{Bild 8: Steuerung des Rechenprozesses}

Iteration wird durch eine Interpolation mit einem Lagrange Polynom beschleunigt und ist in Bild 9 gezeigt.

Wird der Lastfaktor $\lambda$ bei einer Erhöhung der Krümung kleiner, bevor die Zeit vergrossert wird, so ist es nicht mehr möglich, dass die Rechnung konvergiert. In diesem falle handelt es sich um vorzeitiges Versagen infolge Kriechen. 


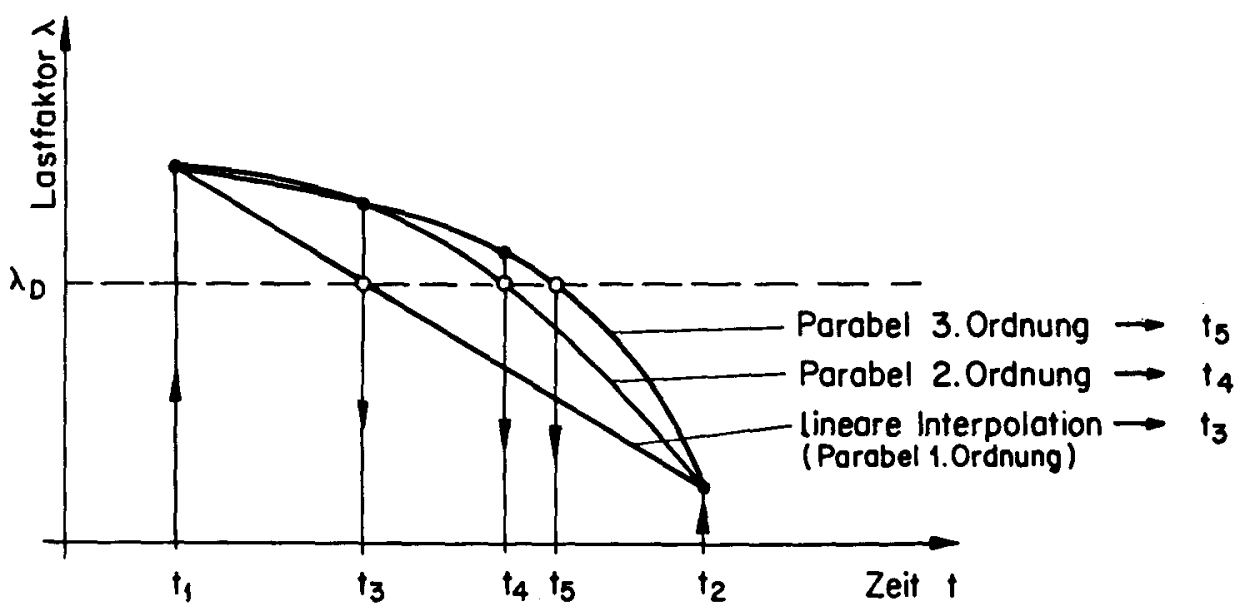

Bild 9: Steuerung für Langzeitlasten: Interpolation von † 


\section{I Genereller Aufbau des Programmes}

Das Programm besteht aus einem Hauptprogramm (MAIN) und 11 Unterprogrammen. Bild 10 gibt einen schematischen Ueberblick über dessen Aufbau.

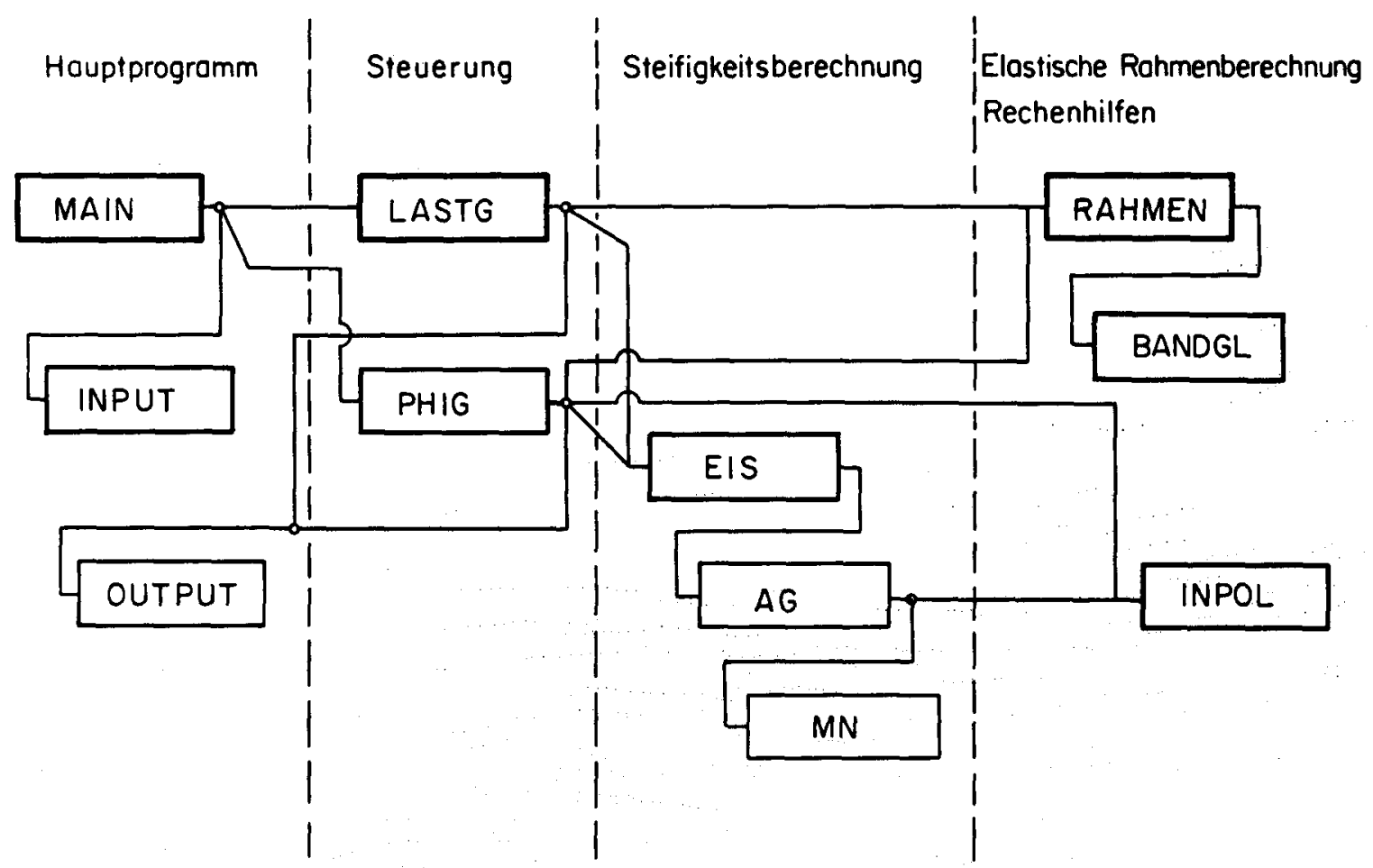

\section{Bild 10: Aufbau des Programmes}

Es können die vier folgenden Gruppen unterschieden werden:

- Hauptprogramm:

- Steuerung:

- Steifigkeitsberechnung:
Zum kurzen Hauptprogramm gehören die Inputund Output-Routinen.

Die Steuerung wird kontrolliert vom Hauptprogramm. LASTG steuert den gegebenen Lastfaktor $\lambda$. PHIG kontrolliert die Krümung $(y / \rho)_{k s}$ im Kontroll-Schnitt bei der Verformungssteuerung.

Die Subroutinen EIS, AG und MN berechnen die Steifigkeit EI für jedes Element unter Berücksichtigung der berechneten Krümmungen und Achsiallasten. (Momenten-Krümmungs-Beziehung) 
- Elastische Rahmenberechnung und Rechenhilfen:
RAHMEN führt eine elastische Rahmenberechnung durch nach der Deformationsmethode unter Berücksichtigung der Theorie 2. Ordnung. BANDGL 18st das dazugehörende lineare Gleichungssystem. INPOL fuhrt eine Interpolation durch mit Hilfe des Polynoms von Langrange nach der Methode von Aitken.

\subsection{Beschrieb der einzelnen Programmteile}

Im folgenden wird eine summarische Beschreibung der einzelnen Programmteile gegeben.

\section{RAHMEN}

Die Biegesteifigkeiten und die Achsiallasten fü jedes Element, die Randbedingungen und alle Lasten müssen gegeben sein. Das Programm berechnet die globale Steifigkeitsmatrix [K] unter Berucksichtigung der Biegesteifigkeiten, der Achsiallasten (Theorie 2. Ordnung) und der Randbedingungen. Für den Lastfaktor $\lambda=1$ und $\lambda=2$ wird je eine elastische Analyse des Tragwerks gemacht. Dabei werden neben den Verschiebungen auch die Stabkrafte und die Reaktionen bestimmt.

Je nach der aktuellen Steuerung werden durch Interpolation die Verschiebungen, Stabkräfte und Reaktionen so ermittelt, dass $\lambda$ mit dem gegebenen Lastfaktor (Laststeuerung), oder dass die Krümung im Kontroll-Schnitt mit der spezifizierten Krummung (Verformungssteuerung) ubereinstimmt.

\section{BANDEL (Bandgleichungen)}

Nach dem Gauss'schen Algorithmus lobt das Programm das lineare Gleichungssystem der Rahmenberechnung, wobei berücksichtigt wird, dass die Steifigkeitsmatrix bandformig und symmetrisch ist.

\section{INPOL (Interpolation)}

Durch n-Stüzstellen wird ein Polynom von $(n-1)$ tem Grade gelegt und die gesuchten Werte werden interpoliert. Bild 9 gibt ein Beispiel fur eine solche Interpolation.

\section{EIS ( Steifigkeit $\overline{\underline{E I}}$ )}

Es werden die Biegesteifigkeiten fü alle Elemente berechnet $\left(\overline{E I}=\bar{M} /\left(y_{p}\right)\right)$. Die inneren Momente $\bar{M}$ werden aufgrund der gegebenen Krümungen $\left(y_{p}\right)$ unter Wahrung des achsialen Gleichgewichts in $A G$ resp. MN ermittelt.

AG (Achsiales Gleichgewicht)

Für eine gegebene Krümmung irgendeines Querschnittes wird die mittlere achsiale Dehnung $\varepsilon_{\mathrm{m}}$ variiert bis das achsiale Gleichgewicht erfullt ist.

Für jede Position der Dehnungsebene werden $\bar{M}$ und $\bar{N}$ in der MN-Routine berechnet. Nach der Durchrechnung von zwei Dehnungspositionen $\varepsilon_{m}$ wird ein neuer Naherungswert fur $\varepsilon_{m}$ durch eine lineare Interpolation bestimmt, sodass sich die dazugehorrende Normalkraft $\overline{\mathrm{N}}$ sehr rasch der gegebenen Normalkraft nahert. Das Konvergenzkriterium kann in der 
INPUT-Routine spezifiziert werden. Hat diese Iteration konvergiert, so wird mit dem entsprechenden $\bar{M}$ in der EIS-Routine die Biegesteifigkeit berechnet.

MN (Moment, Normalkraft)

Für eine gegebene Position der Dehnungsebene werden die inneren Kräfte $\bar{M}$ und $\bar{N}$ durch Integration der Spannungen uber die Querschnittsfläche bestimnt. Die dabei verwendeten $\sigma-\varepsilon-t-$ Beziehungen sind in Kapitel 2.2 beschrieben.

\section{LASTG (Lastgesteuert)}

Der Rahmen wird nach der lastgesteuerten Methode berechnet, die im Kapitel 2.3 ausfühlich beschrieben ist. Eingegeben werden der Lastfaktor $\lambda$ und die Belastungsdauer $\Delta t$.

\section{PHIG (Krümung $\Phi$ wird gesteuert)}

Für Kurzzeitbelastung wird die bezogene Krümmung $\Phi(=H / \rho)$ im Kontroll-Schnitt stufenweise erhöht und der dazugehörende Lastfaktor $\lambda$ wird ermittelt. Ein Versagen infolge Instabilitat stellt sich dann ein, wenn bei einer Erhöhung von $\Phi$ die Last $P$ kleiner wird.

Bei Langzeitbelastung wird zusätzlich noch $\Delta t$ variiert bis $\lambda$ gleich dem gegebenen Faktor $\lambda_{D}$ für die Dauerlast wird. Wird $\lambda$ nach einer Erhöhung der Krümmung kleiner als der gegebene Lastfaktor $\lambda_{D}$ ohne Veränderung von $t$, so bedeutet dies ein vorzeitiges Versagen infolge Kriechen.

\section{INPUT____OUTPEUT}

Diese beiden Routinen sind im Anhang anhand eines Beispieles beschrieben.

\section{MAIIN}

Dieses kurze Programm bestimnt die Steuerungsart gemăss den Input-Daten. 
Die folgenden Beispiele zeigen einige Anwendungsmöglichkeiten des beschriebenen Programmes. Zusätzlich werden einige Resultate mit durchgeführten Versuchen verglichen.

\section{Vergleich mit Versuchen}

Im Rahmen des Forschungsprojektes des Institutes für Baustatik, Abt. Massivbau, ETH Zürich, ưber die Tragfahigkeit von Stahlbetonstutzen wurden verschiedene Stützen unter Kurz- und Langzeitlasten geprüft. Die Resultate dieser Versuche wurden in [5] veroffentlicht. Drei Versuchsstüzen mit verschiedenen Lastgeschichten wurden mit dem beschriebenen Rechenprogramm nachgerechnet. Die drei Stutzen waren beidseitig gelenkig gelagert und hatten dieselbe Lange und denselben Querschnitt. Die Abmessungen sind in Bild 11 gegeben. Infolge der Symmetrie der Lagerung musste nur die Halfte

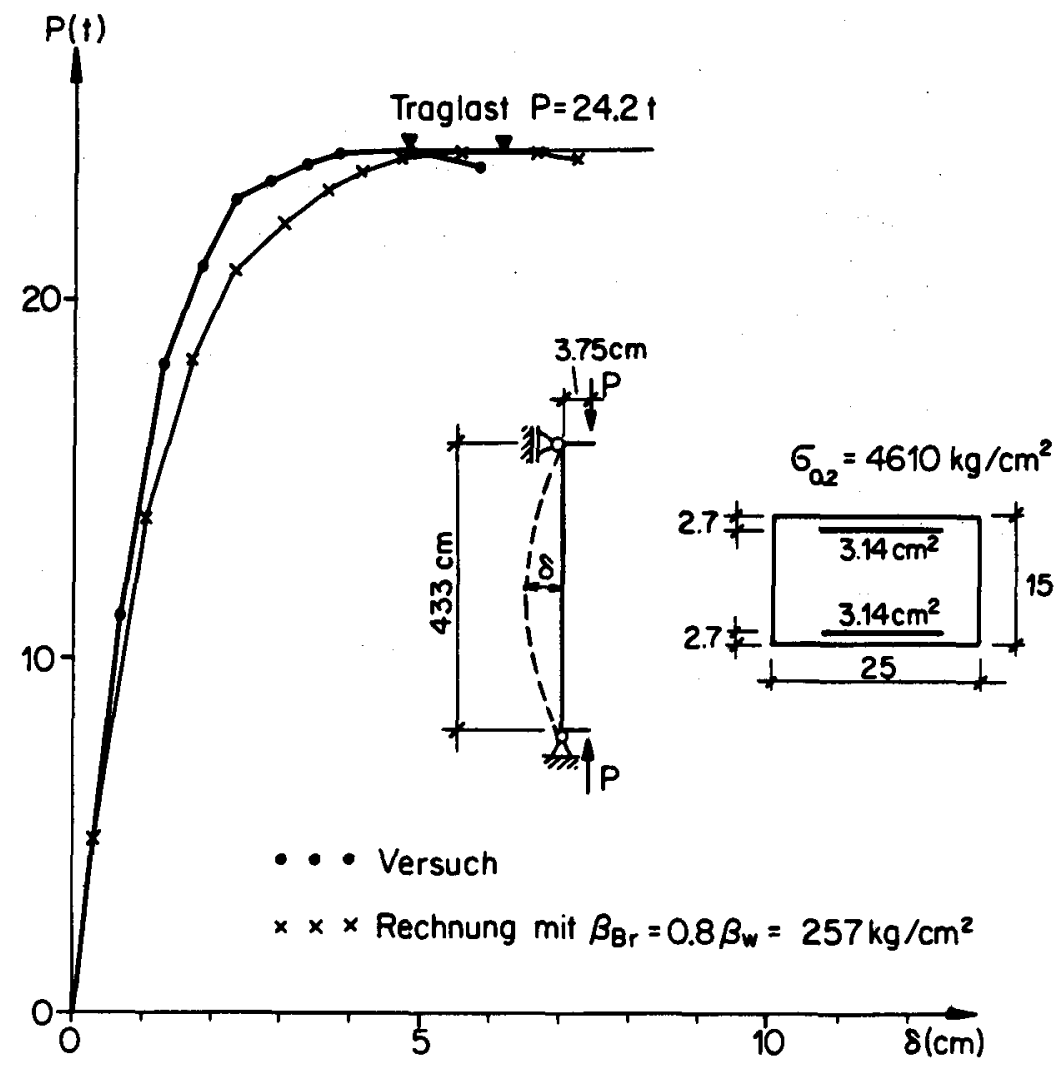

Bild 11: Last - Auslenkungskurve (Stütze 24)

der Stutze gerechnet werden, wobei dieselbe in 6 Elemente eingeteilt wurde. Der Inund Output für Stütze 24 ist im Anhang gegeben.

In einem Kurzzeitversuch wurde die Stütze 24 (Bild 1l) bis zum Bruch belastet. Die gemessene und die berechnete Traglast von 24,2 t stimmen genau uberein. Die Abwei- 
chungen in den mittleren Auslenkungen $\delta$ sind bei der Berechnung auf die Annahmen der $\sigma-\varepsilon$ - Diagramme und die Nichtberücksichtigung der Zugspannungen im Beton zurückzuführen.

Stütze 25 (Bild 12) wurde mit einer Dauerlast $P_{D}=16,4 \mathrm{t}$ während 141 Tagen belastet. Nach dieser Zeit zeigte sich eine eindeutige Tendenz zur Stabilisierung der Verformungen. Auch für diese Stütze ist die Uebereinstimmung der gemessenen und berechneten Traglast im abschliessenden Kurzzeitversuch gut $(<28)$. Die berechneten Auslenkungen sowohl unter Langzeit- wie auch unter Kurzzeitlasten sind aus den oben erwăhnten Gründen etwas zu gross. Die Abhăngigkeit der mittleren Auslenkung $\delta$ von der Zeit ist im unteren Teil des Bildes 12 gezeigt. Die Uebereinstimmung der beiden Kurven ist zufriedenstellend, wenn man berucksichtigt, dass diese Abhänigkeit grossen Streuungen unterworfen ist [6].

Bild 13 zeigt den Langzeitversuch, der zu einem vorzeitigen Versagen infolge Kriechen führte (Stütze 22). Die berechnete Last-Auslenkungskurve stimmt sehr genau mit der gemessenen uberein. Bei einer mittleren Auslenkung $\delta$ von $\mathrm{ca} .9 \mathrm{~cm}$ wird die Stütze unter der konstanten Dauerlast $P_{D}=18,9 t$ instabil. Die Abhăngigkeit der Auslenkung $\delta$ von der Zeit $t$ stimm gut uberein bis kurz vor dem Bruch bei $t=61$ Tagen. Die Rechnung ergibt ein Versagen der Stüze erst bei $t=185$ Tagen. Dieser Zeitpunkt ist aber im Gegensatz zur entsprechenden Auslenkung sehr grossen Streuungen unterworfen. Zur Illustration ist unten im Bild 13 gestrichelt auch der berechnete zeitliche Verlauf von $\delta$ fur eine um 108 niedrigere Bruchspannung $B_{B r}$ des Betons gezeigt. Die Zeitdauer bis zum Kriechbruch ( $t=68$ Tage) wird auf einen Drittel verkürzt.

Abschliessend lässt sich sagen, dass die Last-Auslenkungskurven sich sehr genau berechnen lassen. Hingegen ist der zeitliche Verlauf der Auslenkungen grossen Streuungen unterworfen und kann ohne statistische Untersuchungen nicht zuverlässig berechnet werden. Eine solche Untersuchung wurde in [6] gemacht.

\section{Bogen unter Schneelast}

Es wird ein Bogen berechnet wie er als Binder bei Sporthallen oder Flugzeughangars gebaut werden kann. Das statische System, die Abmessungen und die Lasten sind in Bild 14 gezeigt. Es ist ein beidseits eingespannter Bogen mit einem Zugband, das für die Rechnung durch eine Feder $(k=0,05 \mathrm{~cm} / \mathrm{t})$ ersetzt wird. Der Bogen wird in 14 gerade Elemente eingeteilt.

Als sehr ungüstiger Lastfall wird eine Schneelast von $\lambda \cdot 3.00 \mathrm{t} / \mathrm{m}^{\prime}$ uber die Halfte des Bogens in Rechnung gestellt. Das Eigengewicht $g=3.66 \mathrm{t} / \mathrm{m}^{\prime}$ ist konstant uber die ganze Bogenlänge. Gesucht wird der maximale Lastfaktor $\lambda$ fü die Schneelast. Bild 15 zeigt die berechnete Beziehung zwischen dem Lastfaktor $\lambda$ und der maximalen Durchbiegung $\delta$, die ungefähr im Drittelspunkt unter der Schneelast ermittelt wird. Die gestrichelte Kurve entspricht einer Kurzzeitbelastung. Für die ausgezogene Kurve wird der Bogen zuerst bis zu $\lambda=0,70$ belastet. Diese Last wird wahrend 300 Tagen konstant gehalten und anschliessend bis zur Traglast gesteigert. 


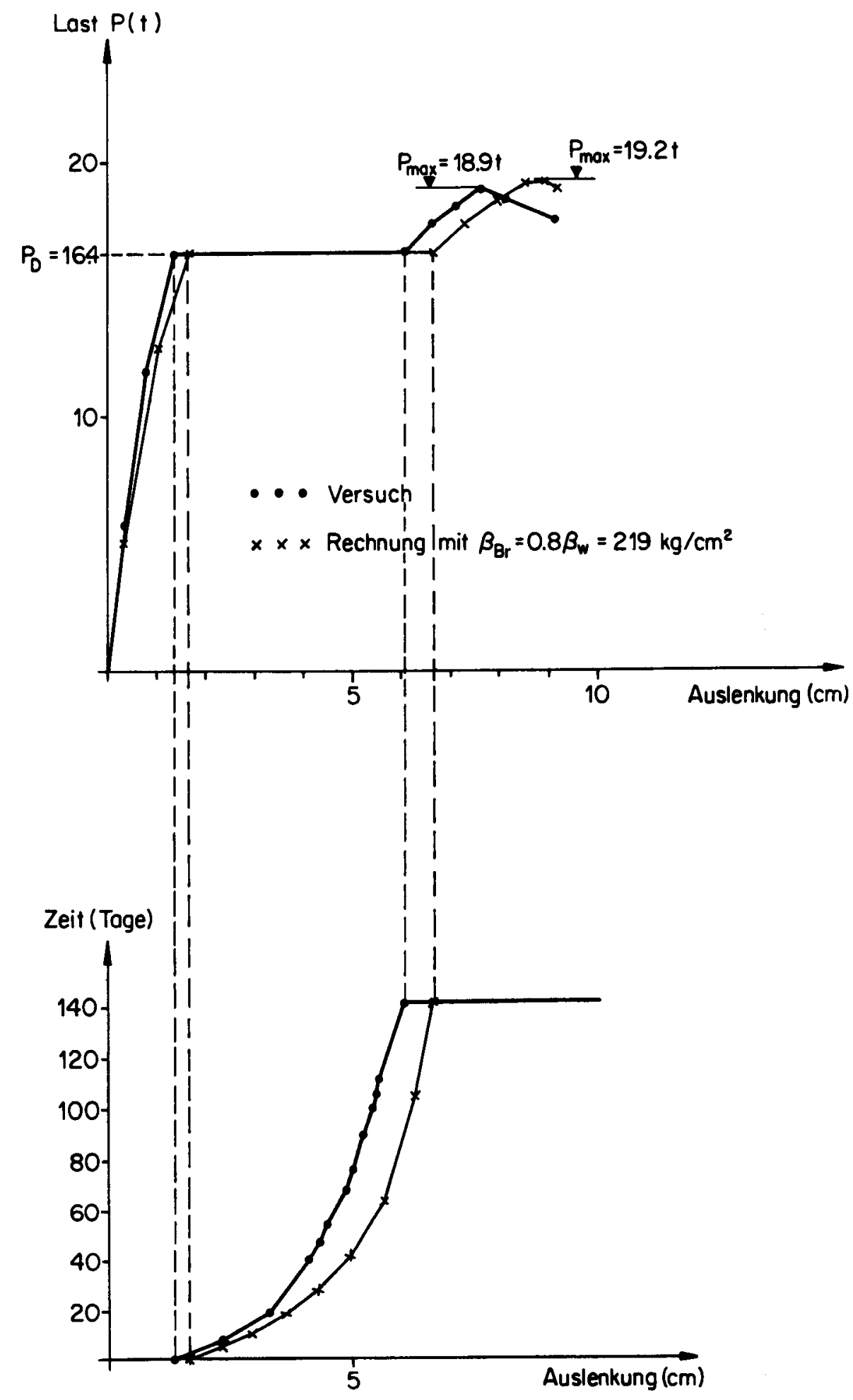

Bild 12: Langzeitversuch mit abschliessendem Kurzzeitversuch (Stütze 25) 


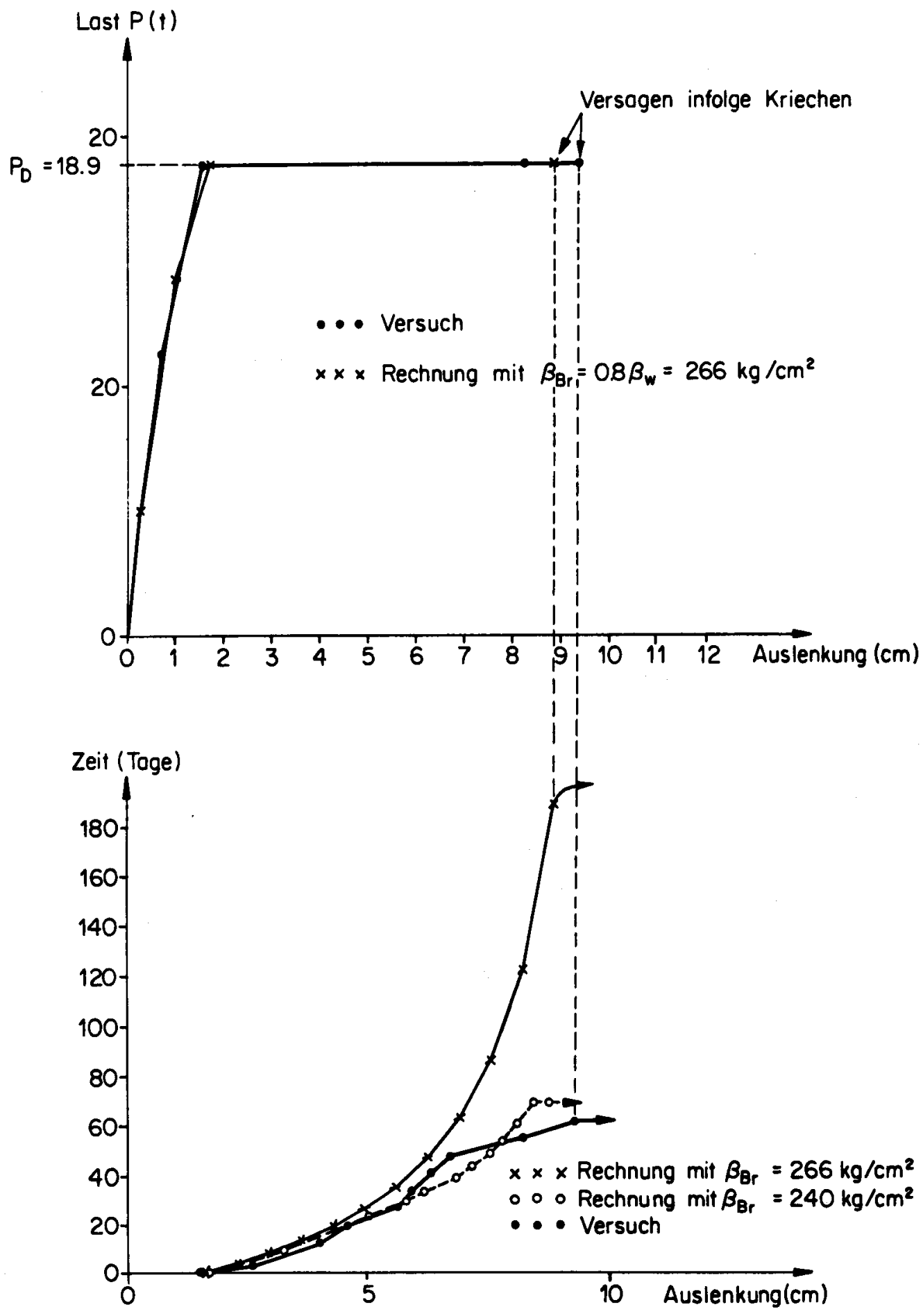

Bild 13: Langzeitversuch bis zum Kriechbruch (Stütze 22) 


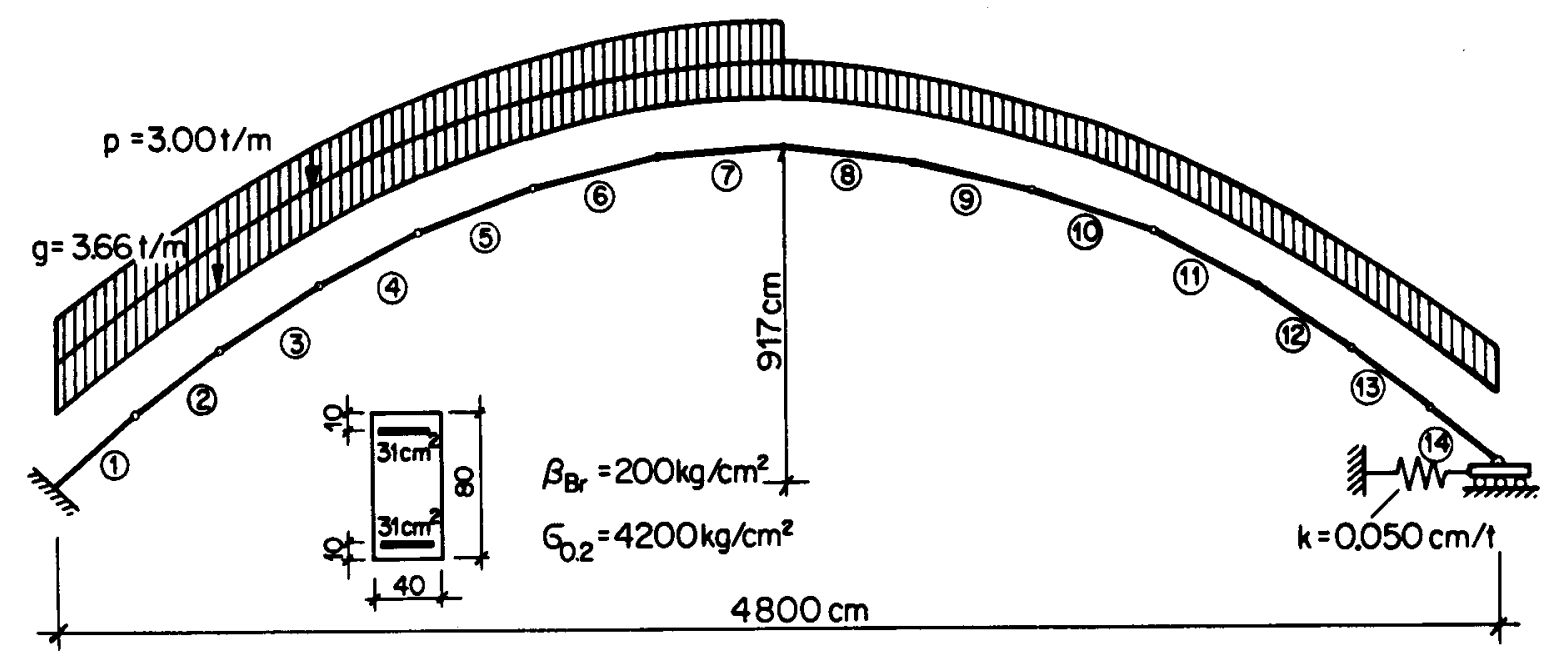

Bild 14: Statisches System und Lasten (Beispiel 2)
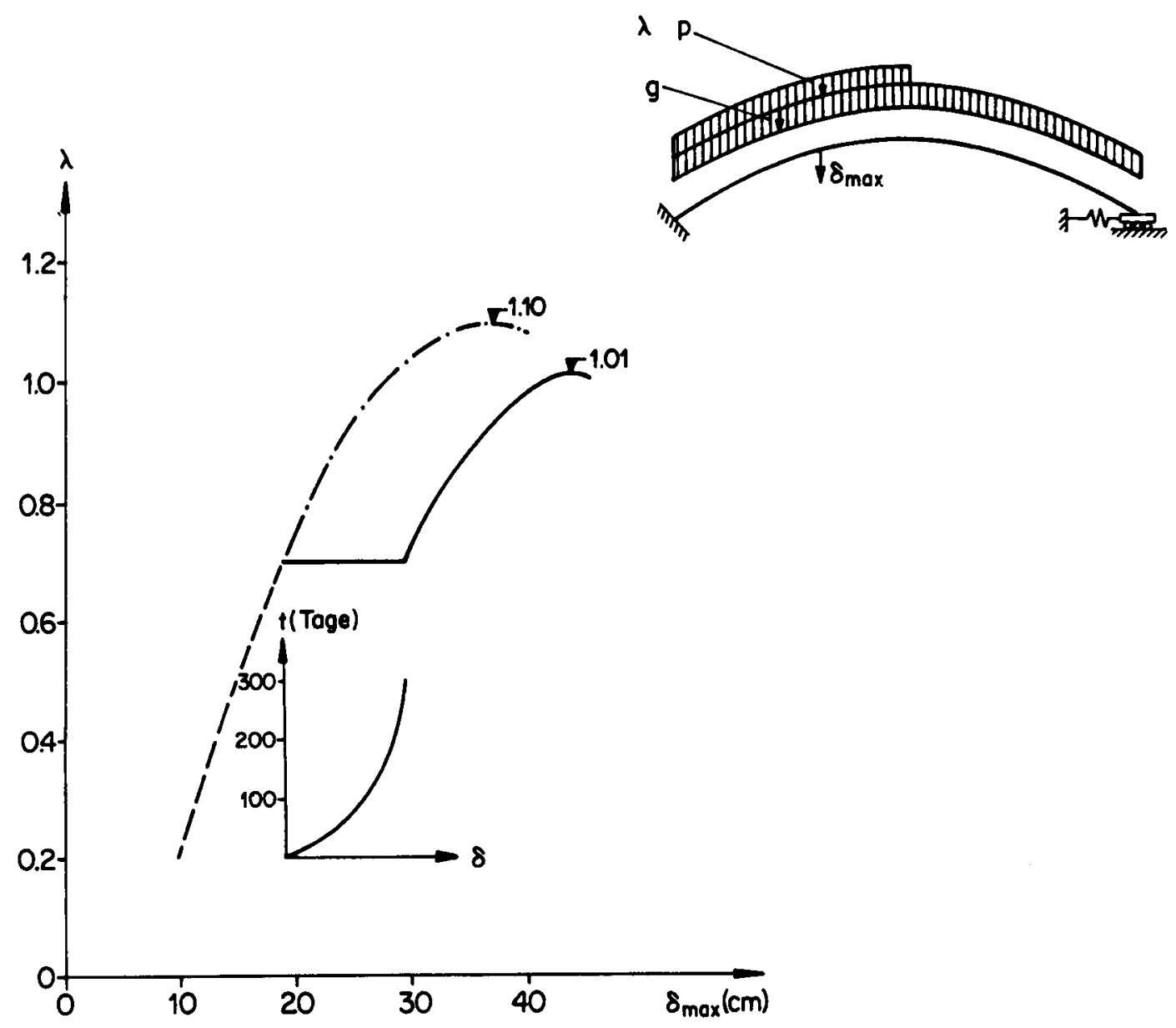

Bild 15: Last-Durchbiegungsverhalten eines Bogens unter Kurz-und Langzeitlasten 


\section{Kräfte, Steifigkeiten}

$\begin{array}{ll}M & \text { ausseres Moment } \\ \bar{M} & \text { inneres Moment } \\ N & \text { Normalkraft } \\ \{P\} & \text { aussere Lasten } \\ P_{D} & \text { Dauerlast } \\ \{R E\} & \text { Reaktionen } \\ {[K]} & \text { Steifigkeitsmatrix des ganzen Tragwerkes } \\ {\left[K_{2}\right]} & \text { Steifigkeitsmatrix nach der Theorie 1. Ordnung } \\ {\left[K_{2}\right]} & \text { Ergänung der Steifigkeitsmatrix infolge der Theorie 2. Ordnung } \\ & \text { (Geometrische Steifigkeitsmatrix) } \\ \overline{E I} & \text { Effektive Biegesteifigkeit }=M /(\gamma \rho) \\ E F & \text { Achsiale Steifigkeit }\end{array}$

\section{Festigkeitswerte, Spannungen}

$\begin{array}{ll}\sigma_{b} & \text { Betonspannung } \\ \beta_{\mathrm{Br}} & \text { Bruchspannung des Betons } \\ \sigma_{S} & \text { Stahlspannung } \\ \sigma_{0,2} & \text { Streckgrenze des Stahles } \\ E_{0} & \text { initialer Elastizitätsmodul } \\ E_{b} & \text { Elastizitätsmodul von Beton } \\ E_{S} & \text { Elastizitätsmodul von Stahl }\end{array}$

Dehnungen, Krümmungen, Verschiebungen, Längen

$\begin{array}{ll}\varepsilon & \text { totale Dehnung } \\ \varepsilon_{m} & \text { achsiale Dehnung in Querschnittsmitte } \\ \varepsilon_{f} & \text { "plastische" Dehnung } \\ \varepsilon_{0} & \text { Dehnung infolge Kurzzeitbelastung } \\ \varepsilon_{s} & \text { Stahldehnung } \\ \varepsilon_{b} & \text { Dehnung bei Erreichen der max. Betonspannung }{ }^{B_{B r}} \\ \varepsilon_{k r} & \text { Kriechdehnung } \\ \varepsilon_{k r, \infty} & \text { Endkriechdehnung } \\ \varepsilon_{\mathrm{sch}} & \text { Schwinddehnung }\end{array}$




$\begin{array}{ll}\varepsilon_{\text {sch, }} & \text { Endschwinddehnung } \\ y_{\rho} & \text { Krümmung } \\ \left(y_{\rho}\right)_{k s} & \text { gegebene Krümmung im Kontroll-Schnitt } \\ \Phi & =H / \rho, \text { bezogene Krümung } \\ \{w\} & \text { Knotenverschiebungen } \\ \text { B } & \text { Querschnittsbreite } \\ \text { H } & \text { Querschnittshohe } \\ \text { L } & \text { Elementlänge }\end{array}$

\section{Verschiedenes}

$\lambda \quad$ Lastfaktor für proportionale Lasten

$\lambda_{D} \quad$ Lastfaktor fur Dauerlasten

K Kriechzahl

$t \quad$ Zeit

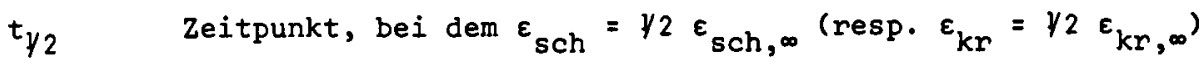

a Drehwinkel eines Elementes gegenuber dem globalen Koordinatensystem

[R] Rotationsmatrix für den Winkel a

U Formanderungsenergie 
[1] Hartz, B.:

"Matrix Formulation of Structural Stability Problems"

ASCE Proc., Vol. 91, ST 5, Dec. 1965

[2] Zienkiewicz, 0.C.:

"The Finite Element Method in Structural and Continuum Mechanics"

McGraw Hill, 1967

[3] Holand, I. and Bell, K.:

"Finite Element Methods in Stress Analysis"

Tapir, Norway, 1969

[4] Fröberg, C.E.:

"Lărobok i numerisk analys"

Svenska Bokforlaget/Bonniers, Stockholm 1962

[5] Ramu, P., Grenacher, M., Baumann, M. und Thurlimann, B.:

"Versuche an gelenkig gelagerten Stahlbetonstutzen unter Dauerlast"

Bericht Nr. 6418-1, Institut für Baustatik, ETH, Zürich, Mai 1969

[6] Oelhafen, U.:

"Formanderungen von Stahlbetonstutzen unter exzentrischer Druckkraft" Bericht Nr. 31, Institut für Baustatik, ETH, Zürich, Okt. 1970 
VERDANKUNGEN

Der vorliegende Bericht wurde im Rahmen eines Forschungsprogrames "Tragfahigkeit von Stahlbetonstützen unter Berlucksichtigung von unelastischen Verformungen" des Instituts für Baustatik, Abt. Massivbau, Eidgenössische Technische Hochschule, Zürich, ausgearbeitet. Dem Leiter dieses Forschungsprogrammes, Herrn Prof. Dr. B. Thürlimann, mochten die Verfasser für die wertvollen Anregungen und Hinweise aufrichtig danken. Die Verfasser schatzten auch die programmtechnischen Ratschläge von Herrn Dr. E. Anderheggen und danken ihm für die Durchsicht des Manuskriptes.

Die Arbeit wurde ermöglicht durch die folgenden grosszligigen Unterstütungen:

Für K. Aas-Jakobsen : The Royal Norwegian Council for Scientific and Industrial Research

und

Johan Helmich Janson og Marcia Jansons Legat.

Für M. Grenacher : Schweizerischer Nationalfonds (Projekt Nr. 4388)

Die Verfasser sind den genannten Stellen zu grossem Dank verpflichtet.

Fur die Gestaltung des Berichtes und die Zeichenarbeiten war Herr G. Göseli verantwortlich. Die Druckbogen wurden von Frl. S. Burki geschrieben. Für ihre Arbeit sei den Genannten bestens gedankt. 
In diesem Bericht wird eine Mタglichkeit aufgezeigt, unelastische Stabtragwerke nach der Theorie 2. Ordnung zu berechnen. Der beschriebene Rechnungsgang basiert auf der Methode der finiten Elemente, wobei die effektiven Biegesteifigkeiten $\overline{\mathrm{EI}}$ für jeden Belastungszustand iterativ aus den wirklichen Momenten-Krümmungs-Beziehungen ermittelt werden. Um die Traglastberechnung zu ermöglichen, wird aus Konvergenzgründen ein verformungsgesteuertes Vorgehen angewendet.

Der Rechenprozess ist für Stahlbetontragwerke beschrieben. Dabei werden auch Langzeiteffekte für kriechen und Schwinden berucksichtigt. Die Kriechverformungen unter variabler Spannung werden mit der "rate of creep" Methode berechnet. Dies wird möglich durch eine Diskretisation der Zeit.

Anhand von zwei Beispielen sind die Möglichkeiten des beschriebenen Rechenverfahrens und des dazugehörenden Computerprogrammes gezeigt.

RESUME

L'objet de ce rapport est la description d'une méthode de calcul selon le $2^{e}$ ordre pour les structures composées de barres inélastique. La méthode des éléments finis est à la base de ce calcul, où les rigidités flexionnelles $\overline{E I}$ sont calculées pour chaque état de charge par iteration à partir des relations moments effectifs-courbures. Pour obtenir la charge de ruine avec une bonne convergence, on doit appliquer au système des déformations.

La méthode est appliquée aux structures de béton armé, et l'on peut y introduire les effets du fluage et du retrait. Les deformations dues au fluage sous une contrainte variable sont calculées par la méthode du "rate of creep", au moyen d'une discrétisation du temps.

On montre à l'aide de deux exemples les possibilités de cette méthode, ainsi que le programme relatif à ce calcul.

SUMMARY

In this report an approach to analyze inelastic frame structures is outlined which considers also the second order effects. The computational process described is based on the finite element method. The effective stiffnesses $\overline{\overline{E I}}$ for each state of loading are determined by trial and error from the actual moment-curvature-relations. To compute the ultimate load and to assure convergence a deformation controlled procedure is employed.

The method is illustrated for reinforced concrete structures. Long time effects such as creep and shrinkage are considered. The deformations due to creep under variable stress are calculated by the "rate of creep method". This is possible by a discretization of time.

Two examples are given to demonstrate the possibilities of the above mentioned analysis and the capability of the computer program which was written for this purpose. 


\section{Eingabe der Daten}

Die Daten werden in der INPUT-Routine mit einer sogenannten Namelist-Anweisung mit dem Namen $D$ (Daten) eingelesen. Jedes Datenpaket besteht aus einer Textkarte und einer beliebigen Anzahl von Datenkarten mit Lochungen nach folgendem Schema:

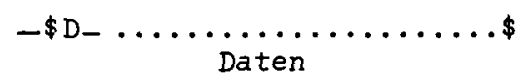

Daten

Die Daten können in beliebiger Reihenfolge eingegeben werden und werden je nach Typ mit dem Parameter SW (Switch) wie folgt geordnet:

$\mathrm{SW}=0$ : Generelle Daten wie Konvergenzkriterien, Kontroll-Schnitt, Materialeigenschaften, Spezifikationen für den Output etc. Einige dieser Grössen (z.B. $\left.E_{s}=2^{\prime} 100^{\prime} 000 \mathrm{~kg} / \mathrm{cm}^{2}\right)$ sind im Programm eingebaut und müssen nur bei Abweichungen eingelesen werden.

SW = 1 : Bedeutet, dass die Daten für eine Tragwerksberechnung alle eingelesen sind und ein neues Problem folgt (vgl. $S W=10)$.

$\mathrm{SW}=2$ : Enthät allgemeine Daten, die im Programm eingebaut sind. Diese Karte muss nicht eingelesen werden.

$S W=3:$ Eingabe der Knoten und Koordinaten. Sind die Knoten auf einer Geraden, dann müssen nur die Endpunkte eingegeben werden.

$S W=4$ : Querschnittsdaten. Die Querschnittsflächen und Fliessspannung und Ueberdeckung der Armierungsstähle können für verschiedene Querschnittstypen eingelesen werden.

$S W=5:$ Zuordnung der Elemente zu den Knoten und Querschnittstypen.

$\mathrm{SW}=6$ : Ort, wo die Biegesteifigkeit $\overline{\mathrm{EI}}$ für ein Element berechnet werden soll. Ohne spezielle Anweisungen wird $\overline{E I}$ in Elementmitte bestimnt. Für das Element mit dem Kontroll-Schnitt wird $\overline{E I}$ in diesem Schnitt bestimmt.

$S W=7$ : Konstante Belastungen und Verschiebungen (Randbedingungen) können in jedem Knoten in allen drei Richtungen gegeben werden.

$\mathrm{SW}=8$ : Proportionale Lasten können in jedem Knoten in allen drei Richtungen gegeben werden. Diese Lasten werden mit dem Lastfaktor $\lambda$ multipliziert.

$S W=9$ : Der Lastfaktor mit der entsprechenden Zeit $t$ kann fü verschiedene Laststufen eingelesen werden. Zusätzlich muss noch die Art der Steuerung eingegeben werden.

$\mathrm{SW}=10$ : Bedeutet, dass die Daten für eine Tragwerksberechnung alle eingelesen sind. Es folgen keine Daten mehr (vgl. $\mathrm{SW}=1$ ).

Ein Beispiel einer solchen Eingabe ist im zweiten Abschnitt gegeben. Wie unter $S W=1$ angedeutet, können gleichzeitig mehrere Probleme eingegeben werden. Das erste Datenpaket muss das Problem vollständig umschreiben mit Ausnahme der im Programm einge- 
bauten Daten. Die folgenden Datenpakete müssen nur die Aenderungen gegenüber dem vorhergehenden Problem oder allfällige Ergänzungen enthalten. Jedes Datenpaket muss aber mit einer Textkarte beginnen. Sämtliche Inputdaten werden vor den entsprechenden Resultaten für jedes Problem ausgeschrieben.

Für die Eingabe der Daten wird die folgende Nomenklatur verwendet:

\begin{tabular}{|c|c|c|c|}
\hline $\begin{array}{l}\text { Verwendet } \\
\text { in } \mathrm{SW}=\end{array}$ & Bezeichnung & $\begin{array}{l}\text { Im Programm } \\
\text { eingebauter } \\
\text { Wert }\end{array}$ & Bedeutung \\
\hline \multirow[t]{14}{*}{0} & $\operatorname{DEL}(1)$ & & $\begin{array}{l}\text { Konvergenzkriterium für } N \text {; } \\
\text { kleinster absoluter Wert }\end{array}$ \\
\hline & $\operatorname{DEL}(2)$ & 0.005 & Relatives Konvergenzkriterium $|\Delta N / N|$ \\
\hline & $\operatorname{DEL}(3)$ & 0.01 & Relatives Konvergenzkriterium $|\Delta \overline{E I} / \overline{E I}|$ \\
\hline & DPHI & 0.0005 & $\begin{array}{l}\text { Schrittgrösse für Krümmung im Kontroll- } \\
\text { Schnitt }\end{array}$ \\
\hline & $E B$ & 0.002 & Betondehnung $\varepsilon_{b}$ für $\sigma_{b}=B_{B r}$ \\
\hline & $\mathrm{EE}$ & 2100000 & Elastizitätsmodul für Stahl \\
\hline & ES & 0.0003 & Endschwinddehrung \\
\hline & IMN & 1 & $\begin{array}{l}\text { 1: Verhalten von Stahlbeton (Langzeit } \\
\text { "Rate of creep") } \\
\text { 2: elastische Analyse }\end{array}$ \\
\hline & IOUT & 1 & $\begin{array}{l}\text { 1: normaler Output } \\
\text { 2: mit Reaktionen, } \overline{E I} \text { und Krümnungen } \\
\text { 3: mit Spannungen } \\
\text { 4: alles }(1 \div 3)\end{array}$ \\
\hline & KS & 1 & Element mit Kontroll-Schnitt \\
\hline & KSE & 1 & Elementende mit Kontroll-Schnitt \\
\hline & RKZ & 2.5 & Endkriechzahl \\
\hline & $S B$ & 200 & Betonbruchspannung ${ }^{B} \mathrm{Br}$ \\
\hline & $\mathrm{TH}$ & 100 & $\begin{array}{l}\text { Zeit in Tagen, nach der die halbe End- } \\
\text { kriechdehnung erreicht ist. }\end{array}$ \\
\hline 3 & $\left.\begin{array}{ll}K 1, & K 2, \\
X 1, & Y 1 \\
X 2, & Y I\end{array}\right\}$ & $\mathrm{DK}=1$ & $\begin{array}{l}\text { Knoten K1 bis } K 2 \text { mit Schritt DK } \\
\text { Koordinaten fur die Knoten KI und K2 }\end{array}$ \\
\hline 4 & $\begin{array}{l}\text { Al, A2 } \\
B, H \\
\text { IQ } \\
\text { SE } \\
\mathrm{U} \\
\end{array}$ & $\begin{array}{r}40 . \\
4200 \\
4 . \\
\end{array}$ & $\begin{array}{l}\text { Armierungsquerschnitte } \\
\text { Breite, Höhe des Betonquerschnittes } \\
\text { Nummer des Querschnittypes } \\
\text { Fliessspannung des Stahles } \\
\text { Ueberdeckung der Armierung }\end{array}$ \\
\hline 5 & $\begin{array}{ll}\mathrm{S} 1, & \mathrm{~S} 2 \\
\mathrm{~K} 1, & \mathrm{~K} 2 \\
\mathrm{IQ} & \end{array}$ & & $\begin{array}{l}\text { Element S1 bis } \mathrm{S} 2 \\
\text { Knotennummern für Element S1 } \\
\text { am Ende } 1 \text { und für Element S2 } \\
\text { am Ende } 2 \\
\text { Zuweisung des Querschnittypes }\end{array}$ \\
\hline 6 & SI & & $\begin{array}{l}\text { Elementnummer } \\
\text { Ort, wo } \overline{E I} \text { bestimmt wird }\end{array}$ \\
\hline 7 & $\begin{array}{lll}\mathrm{K} 1, & \mathrm{~K} 2, & \mathrm{DK} \\
\mathrm{B} 1, & \mathrm{~B} 2, & \mathrm{~B} 3 \\
\mathrm{~V} 1, & \mathrm{~V} 2, & \mathrm{~V} 3 \\
\end{array}$ & $\begin{array}{l}\mathrm{DK}=1 \\
0 . \\
\text { frei }\end{array}$ & $\begin{array}{l}\text { Knoten } \mathrm{K} 1 \text { bis } \mathrm{K} 2 \text { mit Schritt DK } \\
\text { Belastung in } \mathrm{x}-, \mathrm{y}-\text { und } a-\text { Richtung } \\
\text { Verschiebung in } x-, y-\text { und } \alpha-\text { Richtung }\end{array}$ \\
\hline 8 & $\begin{array}{lll}\mathrm{K} 1, & \mathrm{~K} 2, & \mathrm{DK} \\
\mathrm{B} 1, & \mathrm{~B} 2, & \mathrm{~B} 3\end{array}$ & $D K=1$ & $\begin{array}{l}\text { Knoten K1 bis } K 2 \text { mit Schritt DK } \\
\text { Wert der proportionalen Lasten für } \lambda=1.0\end{array}$ \\
\hline
\end{tabular}




\begin{tabular}{|c|c|c|c|}
\hline $\begin{array}{l}\text { Verwendet } \\
\text { in } S W=\end{array}$ & Bezeichnung & $\begin{array}{l}\text { Im Programm } \\
\text { eingebauter } \\
\text { Wert }\end{array}$ & Bedeutung \\
\hline 9 & $\begin{array}{l}\text { IT } \\
\text { L } \\
\text { LS } \\
\text { T }\end{array}$ & $\begin{array}{l}1 \\
1 \\
0\end{array}$ & $\begin{array}{l}\text { Art der Steuerung } \\
\text { 1. Laststeuerung } \\
\text { 2. Krummungssteuerung } \\
\text { Lastfaktor } \lambda \\
\text { Laststufe } \\
\text { Zeit }\end{array}$ \\
\hline
\end{tabular}

\section{Beispiel einer Dateneingabe}

Es wird die Eingabe für das Beispiel Nr. 1 in Kap. 3 gegeben. Mit den oben gegebenen Bezeichnungen sieht die Eingabe wie folgt aus:

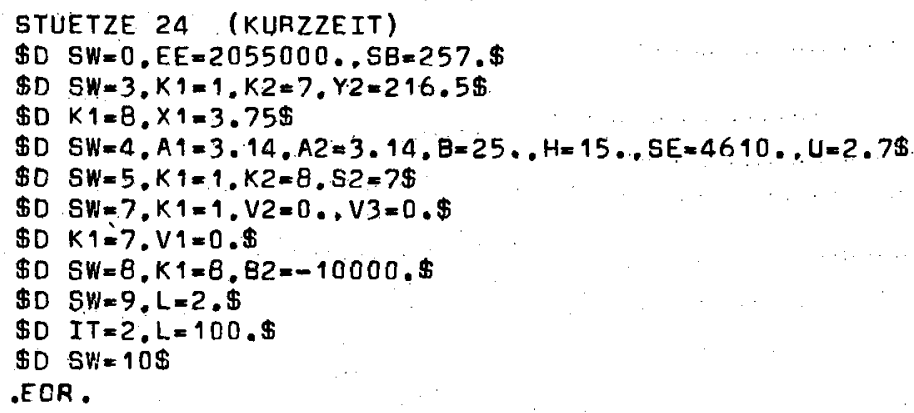

\section{Output der Resultate}

In normalen Output werden für alle Elemente die Normalkräte, die Momente an beiden Enden, die Verschiebungen senkrecht zu den Elementachsen und die Dehnungen der Zugund Druckarmierung ausgedruckt. Dazu wird die Krümmung PHI im Kontroll-Schnitt, der Lastfaktor LFT und die Zeit gegeben. Zur Kontrolle wird nach jedem Output die Anzahl der durchgeführten elastischen Analysen (ITER), die Rechenzeit ( $T$ ), der Steuerungstyp (TYP) und das verwendete Materialverhalten (IMN) ausgeschrieben. Mit entsprechenden Befehlen können auch die Biegesteifigkeiten, Krümmungen und die Spannungsverteilung über den Querschnitt fü alle Elemente sowie die Reaktionen verlangt werden. Als Illustration folgt der Output für Beispiel Nr. I: 
STUETZE 24 (KURZZEIT)

MATERIALEIGENSCHAFTEN:

$\begin{array}{rrrrrr}\text { EME } & \text { SB } & E B & K Z & T / 2 & \text { ES } \\ 2055000 & 257 & .0020 & 2.50 & 100 & .0003\end{array}$

QUERSCHNITTE:

$\begin{array}{ccccccr}\text { Q } & \text { B } & H & \text { U } & \text { A1 } & \text { A2 } & \text { SE } \\ 1 & 25.0 & 15.0 & 2.70 & 3.140 & 3.140 & 4610\end{array}$

STAEBE:

$\begin{array}{rrrrrrrrrr}S & K 1 & K 2 & 0 & X 1 & Y 1 & X P & Y P & \text { SI } & T Y \\ 1 & 1 & 2 & 1 & 0.00 & 0.00 & 0.00 & 36.08 & 0.00 & 1 \\ 2 & 2 & 3 & 1 & 0.00 & 36.08 & 0.00 & 72.17 & .50 & 1 \\ 3 & 3 & 4 & 1 & 0.00 & 72.17 & 0.00 & 108.25 & .50 & 1 \\ 4 & 4 & 5 & 1 & 0.00 & 108.25 & 0.00 & 144.33 & .50 & 1 \\ 5 & 5 & 6 & 1 & 0.00 & 144.33 & 0.00 & 180.42 & .50 & 1 \\ 6 & 6 & 7 & 1 & 0.00 & 180.42 & 0.00 & 216.50 & .50 & 1 \\ 7 & 7 & 8 & 1 & 0.00 & 216.50 & 3.75 & 216.50 & .50 & 1\end{array}$

KONSTANTE LASTEN/VERSCHIEBINNGEN:

$\begin{array}{rrrrrrr}K & B 9 & 82 & B 3 & V 1 & V 2 & V 3 \\ 1 & 0 & 0 & 0 & * 000.000 & 0.000 & 0.000 \\ 7 & 0 & 0 & 0 & 0.000 & * 000.000 & * 000.000\end{array}$

PROPORTIONALE LASTEN:

$\begin{array}{rrrr}K & B 1 & \text { B2 } & \text { B3 } \\ B & 0 & -1000 & 0\end{array}$

LASTFAKTOR/ZEIT:

\begin{tabular}{rrr} 
TYP & LASTF & \multicolumn{1}{c}{ ZEIT } \\
1 & 2.00 & 0 \\
2 & 100.00 & 0
\end{tabular}

KONVEAGENZ-KRITERIEN:

DN DN/N DEI/EI

$100 \quad .005 \quad .010$

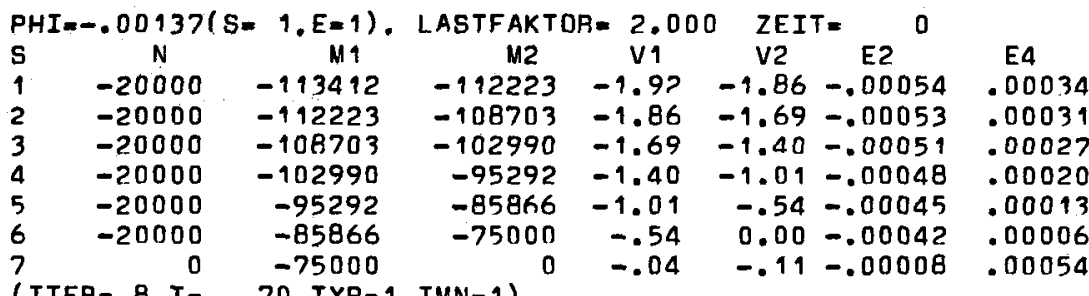

(ITEA $=8 . T=.70, T Y P=1, I M N=1$ )

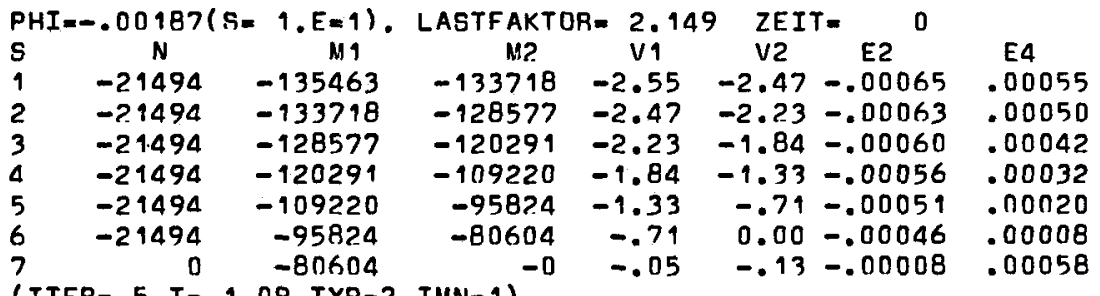

(ITER $=5 . T=1.09$, TYP $=2$, IMN $=1$ ) 


\begin{tabular}{|c|c|c|c|c|c|c|c|}
\hline & .00237 & १. $E=1)$, & LASTFAK & $\theta=2$. & ZFI & $T=$ & \\
\hline 5 & & $\begin{array}{c}M 1 \\
-156362\end{array}$ & 112 & V1 & V2 & F? & F4 \\
\hline $\begin{array}{l}1 \\
2\end{array}$ & $\begin{array}{l}-2.2587 \\
-2.2587\end{array}$ & $\begin{array}{l}-156362 \\
-154041\end{array}$ & -154041 & -3.17 & $\begin{array}{l}-3.07 \\
-2.77\end{array}$ & -.00075 & $\begin{array}{l}.00077 \\
.00070\end{array}$ \\
\hline 3 & -22587 & -147213 & -136253 & -2.77 & -2.28 & -.00069 & $.0005 \mathrm{~B}$ \\
\hline 4 & -22587 & -136253 & -121723 & -2.28 & -1.64 & $-.0 \cap 063$ & $.0 n 04$ \\
\hline 5 & -27587 & -121723 & $-1043 n 0$ & -1.64 & -.87 & -.00056 & .00026 \\
\hline 6 & -22587 & -104300 & -84703 & -.87 & 0.00 & -.00049 & . 0 ก \\
\hline 7 & & $-847 n 3$ & -0 & -.05 & -.15 & -.00009 & .0006 \\
\hline
\end{tabular}

\begin{tabular}{|c|c|c|c|c|c|c|c|}
\hline & & & & & ZEIT & 0 & \\
\hline$s$ & N & $M 19$ & M? & $v_{1}$ & v? & F? & F4 \\
\hline 1 & -23300 & -175774 & -172876 & -3.79 & -3.67 &.$- \cap ก \cap 85$ & . $n \cap \cap 99$ \\
\hline ? & -2.3302 & -172876 & -164366 & -3.67 & -3.30 & $-.0008 ?$ & . חกחRg \\
\hline 3 & -23302 & -164366 & $-15073 A$ & & -2.72 & -.00076 & .00075 \\
\hline$\Delta$ & -73302 & -150738 & -132746 & -2.72 & -1.95 &.$- n n 069$ & .00054 \\
\hline 5 & -23302 & -132.746 & -111309 & -1.95 & -1.03 & $-.0 \cap n 61$ & .00033 \\
\hline A & $-2330 ?$ & -111309 & -87382 & -1.03 & 0.00 & -.00052 & .00012 \\
\hline 7 & & -87382 & 0 & -.05 & -.16 & -.00009 & .00063 \\
\hline
\end{tabular}

\begin{tabular}{|c|c|c|c|c|c|c|c|}
\hline & 033 & $1, F=11$. & & a. & 7.FI & $T=$ & \\
\hline 7 & $N$ & $M 1$ & M? & $1 / 9$ & V? & F2 & FA \\
\hline 1 & -2.3770 & -193815 & -190349 & -4.41 & -4.26 & .00095 & .00121 \\
\hline$?$ & & & & & & & ח \\
\hline 3 & -23770 & -180181 & -16 & -3. & -3.15 & 0084 & .00092 \\
\hline 4 & -2.3770 & -163940 & -142568 & -3.15 & -2.25 & -.00075 & .00067 \\
\hline 5 & & -142 & -19 & -2. & -1.18 & -.000 & \\
\hline 6 & -23770 & -117230 & -89137 & -1.18 & 0.00 & -.00053 & .00014 \\
\hline 7 & & -89137 & $-n$ & -.05 & -.18 & -.00009 & .00064 \\
\hline
\end{tabular}

\begin{tabular}{|c|c|c|c|c|c|c|c|}
\hline & .00387 & $1, E=1)$ & LASTFAK & $9=2$. & $7 F I$ & $T=$ & \\
\hline ล & $\mathrm{N}$ & M1 & M2 & $1 / 1$ & V? & F? & F4 \\
\hline 1 & -23989 & -210312 & -2062.83 & -5.09 & -4.84 & -.00104 & .00143 \\
\hline 2 & -23989 & -206283 & -194490 & -4.84 & & 099 & ก129 \\
\hline 3 & -2.3989 & -194490 & -175707 & -4.35 & -3.57 & -.00091 & .00108 \\
\hline 4 & $\begin{array}{l}-23989 \\
23090\end{array}$ & -175707 & -151074 & -3.57 & -2.54 & -.00080 & .00079 \\
\hline 6 & $\begin{array}{l}-23989 \\
-23989\end{array}$ & -122006 & -89958 & $\begin{array}{l}-2.54 \\
-9.33\end{array}$ & $\begin{array}{r}-1.33 \\
0.00\end{array}$ & $\begin{array}{l}-.00068 \\
-.00055\end{array}$ & .00095 \\
\hline$?$ & & -89958 & -0 & -.05 & -.20 & -.00009 & .00065 \\
\hline
\end{tabular}

( ITER $=4 . T=2.38, T Y P=2 . I M N=1$ )

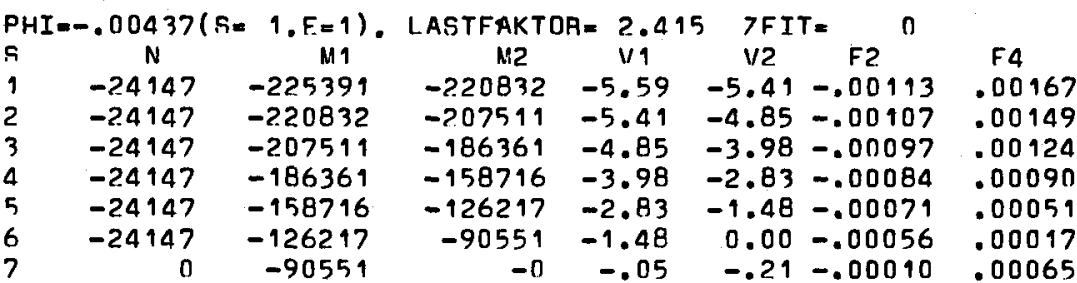

(ITER $=4, T=2.67, T Y P=2, I M N=1$ )

\begin{tabular}{|c|c|c|c|c|c|c|c|}
\hline & 1048 & $t=11$. & Ir & 2.4 & ZEIT: & 0 & \\
\hline s & $\mathrm{N}$ & $M 1$ & M2. & $v_{1}$ & V? & $F$ ? & F4 \\
\hline 9 & -24163 & 39771 & -234691 & -6.19 & -5.98 & .00123 & .00989 \\
\hline 2 & -24963 & -234691 & 9861 & -5.98 & & -.001 & .00169 \\
\hline 3 & -24163 & 1861 & & & & & \\
\hline & -24163 & 367 & 747 & -4.39 & -3.12 & -.000 & \\
\hline & -24163 & 747 & & & -1.63 & & \\
\hline 6 & -24163 & -129858 & -90611 & -1.63 & 0.00 & -.0 & \\
\hline$?$ & $=0$ & $\begin{array}{c}-90611 \\
89 . T Y P=2\end{array}$ & $I M N=1)^{-0}$ & 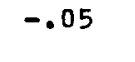 & & -.00010 & \\
\hline PH & 0053715 & 1. $E=1)$. & LASTFAKTOR & 2.408 & 7EIT: & 0 & \\
\hline $\mathrm{s}$ & & N11 & M2 & vi & v? & $\mathrm{F}$ ? & F4 \\
\hline 1 & -2.4083 & -2.52904 & -247318 & -6.76 & -6.53 & -.00132 & .00212 \\
\hline 2 & -2.4083 & 7318 & -231042 & -6. & & -.0012 & .00489 \\
\hline 3 & -24083 & -231042 & $-20532 ?$ & -5.8 & -4.78 & -.0010 & .00 \\
\hline 4 & -24083 & $-20532 ?$ & -171904 & -4.78 & -3.39 & -.00092 & .00143 \\
\hline 5 & -24083 & -171904 & -132862 & -3.3 & -1.77 & -.00075 & \\
\hline 6 & -24083 & $-13286 ?$ & -90311 & -1.77 & 0.00 & -.00057 & .00 \\
\hline & $=3 . T^{0}=$ & -90311 & )$^{-0}$ & & & & \\
\hline
\end{tabular}

